Journal for ImmunoTherapy of Cancer

\title{
Interrupting the nitrosative stress fuels tumor-specific cytotoxic T lymphocytes in pancreatic cancer
}

Francesco De Sanctis, ${ }^{1}$ Alessia Lamolinara, ${ }^{2}$ Federico Boschi, ${ }^{3}$ Chiara Musiu (D),${ }^{1}$ Simone Caligola, ${ }^{1}$ Rosalinda Trovato, ${ }^{1}$ Alessandra Fiore, ${ }^{1}$ Cristina Frusteri, ${ }^{1}$ Cristina Anselmi, ${ }^{1}$ Ornella Poffe, ${ }^{1}$ Tiziana Cestari, ${ }^{1}$ Stefania Canè, ${ }^{1}$ Silvia Sartoris, ${ }^{1}$ Rosalba Giugno, ${ }^{3}$ Giulia Del Rosario, ${ }^{4}$ Barbara Zappacosta, ${ }^{5}$ Francesco Del Pizzo, ${ }^{2}$ Matteo Fassan, ${ }^{6,7}$ Erica Dugnani, ${ }^{8}$ Lorenzo Piemonti, ${ }^{8,9}$ Emanuela Bottani, ${ }^{10}$ Ilaria Decimo, ${ }^{10}$ Salvatore Paiella, ${ }^{11}$ Roberto Salvia, ${ }^{11}$ Rita Teresa Lawlor, ${ }^{12}$ Vincenzo Corbo, ${ }^{12,13}$ Youngkyu Park, ${ }^{14,15}$ David A Tuveson, ${ }^{14,15}$ Claudio Bassi, ${ }^{11}$ Aldo Scarpa, ${ }^{12,13}$ Manuela lezzi, ${ }^{2}$ Stefano Ugel, ${ }^{1}$ Vincenzo Bronte (D) ${ }^{1}$

To cite: De Sanctis $F$, Lamolinara A, Boschi F, et al. Interrupting the nitrosative stress fuels tumor-specific cytotoxic T lymphocytes in pancreatic cancer. Journal for ImmunoTherapy of Cancer 2022;10:e003549. doi:10.1136/ jitc-2021-003549

- Additional supplemental material is published online only. To view, please visit the journal online (http://dx.doi.org/10. 1136/jitc-2021-003549).

SU and VB are joint senior authors.

Accepted 04 December 2021

Check for updates

(c) Author(s) (or their employer(s)) 2022. Re-use permitted under CC BY-NC. No commercial re-use. See rights and permissions. Published by BMJ.

For numbered affiliations see end of article.

\section{Correspondence to} Professor Vincenzo Bronte; vincenzo.bronte@univr.it

Dr Francesco De Sanctis; francesco.desanctis@univr.it

\section{ABSTRACT}

Background Pancreatic ductal adenocarcinoma (PDAC) is one of the deadliest tumors owing to its robust desmoplasia, low immunogenicity, and recruitment of cancer-conditioned, immunoregulatory myeloid cells. These features strongly limit the success of immunotherapy as a single agent, thereby suggesting the need for the development of a multitargeted approach. The goal is to foster T lymphocyte infiltration within the tumor landscape and neutralize cancer-triggered immune suppression, to enhance the therapeutic effectiveness of immune-based treatments, such as anticancer adoptive cell therapy (ACT).

Methods We examined the contribution of immunosuppressive myeloid cells expressing arginase 1 and nitric oxide synthase 2 in building up a reactive nitrogen species (RNS)-dependent chemical barrier and shaping the PDAC immune landscape. We examined the impact of pharmacological RNS interference on overcoming the recruitment and immunosuppressive activity of tumor-expanded myeloid cells, which render pancreatic cancers resistant to immunotherapy. Results PDAC progression is marked by a stepwise infiltration of myeloid cells, which enforces a highly immunosuppressive microenvironment through the uncontrolled metabolism of L-arginine by arginase 1 and inducible nitric oxide synthase activity, resulting in the production of large amounts of reactive oxygen and nitrogen species. The extensive accumulation of myeloid suppressing cells and nitrated tyrosines (nitrotyrosine, $\mathrm{N}$-Ty) establishes an RNS-dependent chemical barrier that impairs tumor infiltration by T lymphocytes and restricts the efficacy of adoptive immunotherapy. $A$ pharmacological treatment with AT38 ([3-(aminocarbonyl) furoxan-4-yl]methyl salicylate) reprograms the tumor microenvironment from protumoral to antitumoral, which supports T lymphocyte entrance within the tumor core and aids the efficacy of ACT with telomerase-specific cytotoxic T lymphocytes.

Conclusions Tumor microenvironment reprogramming by ablating aberrant RNS production bypasses the current limits of immunotherapy in PDAC by overcoming immune resistance.

\section{INTRODUCTION}

Pancreatic ductal adenocarcinoma (PDAC) is a late stage-diagnosed, treatment orphaned disease that is characterized by a dismal $10 \%$ overall survival at 5 years. The poor progress in early-diagnosis biomarker identification and therapeutic options will soon make this malignancy the second leading cause of cancer-related deaths. ${ }^{1}$ Furthermore, immunotherapy approaches, such as immune checkpoint inhibitor-based therapies, do not provide any benefit when used as a single agent ${ }^{2}$ mainly because of the intrinsic low immunogenicity ${ }^{3}$ and the establishment of a powerful immunosuppressive tumor microenvironment (TME). ${ }^{4}$ Indeed, PDAC has a low mutation load, with almost complete absence of tumor-specific neoepitopes in both human specimens and mouse preclinical models, ${ }^{5}$ unlike immunotherapyresponsive cancers. ${ }^{6}$ In contrast, pancreatic cancer expresses several tumor-associated antigens (TAAs), such as mesothelin, enolase, telomerase (TERT), carcinoembryonic antigen and Wilms' tumor gene $1 .^{7}$ TERT is ranked as one of the most prioritized TAAs, as its expression is shared in tumors with different histology, in all evolutionary phases of tumor progression and cell subsets, including metastatic and cancer stem cells. ${ }^{8}$ TERT is an enzyme that replaces the ends of chromosomes during DNA replication and prevents cell senescence. For this reason, TERT is enlisted as a key tumor 
hallmark $^{89}$ and several TERT-targeting and anticancer vaccination protocols have been developed in different tumor settings. Nonetheless, most of these approaches do not improve survival of patient with cancer. The failure can be mainly attributed to the limited endogenous repertoire of $\mathrm{T}$ lymphocytes specific for self-proteins, which are constrained and deleted during thymus development, thereby generating a pool of lymphocytes with low avidity $\mathrm{T}$ cell receptors (TCRs). Adoptive immunotherapy can overcome these limitations. Mouse (H-2 $\mathrm{K}^{\mathrm{b}}$-restricted TERT ${ }_{198-205}$-specific), ${ }^{10}$ or human (HLA-A2restricted TERT ${ }_{\left.865-873^{-s p e c i f i c}\right)^{11}}$ cytotoxic $\mathrm{T}$ lymphocyte (CTL)-based adoptive cell therapy (ACT) restricts tumor progression in both hematological and solid cancers in mouse and human preclinical settings, respectively, ${ }^{11-13}$ thereby justifying the application of TERT-targeting adoptive immunotherapy for a large fraction of patients.

Pancreatic cancer progression establishes a 'cold' microenvironment where $\mathrm{T}$ lymphocytes are limited to the tumor border and exhibit low expression of activation markers. ${ }^{414}$ These features delineate a state of immunological ignorance, which can underlie both primary and adaptive immunotherapy resistance. ${ }^{15}$ Tumor hijacks both fibroblasts and myeloid cells to promote its outgrowth and sustain a state of immune tolerance. High extracellular matrix (ECM) deposition, triggered by cancer-associated fibroblasts (CAFs), along with progressive myeloid-derived suppressor cell (MDSC) and tumor-associated macrophage (TAM) infiltration can restrain the homing of $\mathrm{T}$ lymphocytes to the tumor core. ${ }^{16}{ }^{17}$ Cancer-associated myeloid cells abrogate adaptive $\mathrm{T}$ lymphocyte-based responses by many mechanisms, ${ }^{17}$ including synergic expression of arginase-1 (ARG1) and inducible nitric oxide synthase (iNOS/NOS2), which support reactive nitrogen species (RNS) generation. These hyper-reactive molecules are responsible for altering protein structure by promoting post-translational modifications, such as 3-nitrotyrosine (N-Ty) induction, a consistent marker of RNS production, which in turn critically affects protein-protein interactions and functions, including homing of T lymphocytes to tumors, antigen recognition and T lymphocyte activation. ${ }^{18}$ Therefore, the targeting of RNS-dependent immune alterations in TME is a relevant goal to support immunotherapy effectiveness in PDAC.

In the present study, we validated TERT expression in PDAC and clarified how the organization of an immunosuppressive TME that assists N-Ty accumulation can limit the homing of T lymphocytes to tumors in human PDAC, as well as in transplantable and genetically engineered mouse pancreatic cancer models (ie, H-2 ${ }^{\mathrm{b}}, \mathrm{Kras}^{\mathrm{LSL-G12D/+}}$; $\left.\operatorname{Trp} 53^{L S L-R 172 H /+} ; \mathrm{Pdx}-1-C r e^{-/+} ; \mathrm{KPC}\right)$. Moreover, we unveiled how TME can be converted from a protumoral to an antitumoral milieu by AT38 ((3-(aminocarbonyl)furoxan4-yl)methyl salicylate) administration, ${ }^{19}$ which impairs RNS-based barrier by interfering with the expression of ARG1 and NOS2 enzymes in myeloid cells. Finally, we investigated whether the abrogation of RNS-dependent hurdles can unleash the antitumor effect of TERT-specific ACT in the context of PDAC.

\section{METHODS}

\section{Animal studies}

Eight-week-old $\mathrm{C} 57 \mathrm{Bl} / 6 \mathrm{~J}\left(\mathrm{H}-2^{\mathrm{b}}\right.$, referred as wild type, WT) mice were purchased from Charles River Laboratories Inc (Calco, Italy); NOG mice (NOD.Cg-Prkdc $c^{\text {scid }} \mathrm{Il}$ $2 r g^{t m I S u g} / \mathrm{JicTac}$ ) were purchased from Taconic (Hudson, New York, USA); B6.SJL-Ptprc ${ }^{a} P e p c^{b} /$ BoyJ (H-2 ${ }^{b}$, referred as CD45.1) congenic mice and TCR-transgenic mice $\mathrm{C} 57 \mathrm{BL} / 6-\mathrm{Tg}$ (TcraTcrb) $1100 \mathrm{Mjb} / \mathrm{J} \quad\left(\mathrm{H}-2^{\mathrm{b}}\right.$, referred as OT-1) were purchased from Jackson Laboratories (Bar Harbor, Maine, USA). KPC mice were kindly supplied by Dr D Tuveson (Cold Spring Harbor Laboratory, New York, USA). ${ }^{5}$ All mice were maintained under specific pathogen-free conditions in the animal facility of the University of Verona. Food and water were provided ad libitum. All genetically transgenic mice and their respective controls were gender and age-matched (typically 8-10 weeks) and both males and females were used in this study. Animal experiments were performed according to national (protocol number C46F4.1 approved by the Ministerial Decree Number 1044/2016-PR of October 26, 2016 and protocol number C46F4.8 approved by the Ministerial Decree Number 207/2018-PR of March 14, 2018) and Europeans laws and regulations. All animal experiments were approved by Verona University Ethical Committee (http://www.medicina.univr.it/fol/main? ent=bibliocr\&id=85) and conducted according to the guidelines of Federation of European Laboratory Animal Science Association (FELASA). All animal experiments were in accordance with the Amsterdam Protocol on animal protection and welfare: mice were monitored daily and euthanized when displaying excessive discomfort.

\section{Human samples}

Tissue specimens and clinical data of two independent PDAC patient cohorts were retrieved from the ARC-Net Biobank at Verona University Hospital (https://arcnetit. wordpress.com/biobanca/). All patients gave informed consent to biobank prior the study (Prot. 52070, Prog. 1885 on $17 / 11 / 2010$, principal investigator (PI): A.S.) and the study was approved by the Ethics Committee (Prot. 25978, Prog. 2172 on 29/05/2012, PI: A.S.). The clinical features of PDAC subjects involved in the study are recapitulated in tables 1 and 2.

\section{Statistical analysis}

Comparison between two groups were performed using an unpaired two-tailed Mann-Whitney $U$-test (unpaired samples), a paired two-tailed Mann-Whitney $U$-test (paired samples), and a two-tailed Student's $t$-test (normally distributed parameters). Multiple samples were compared using analysis of variance with Turkey's posttest for multiple comparisons. Correlations were analyzed with Spearman's rank correlation. Survival curves were 
Table 1 Clinical features of 88 treatment-naïve patients with PDAC involved in the study of TERT antigen expression

\begin{tabular}{lll}
\hline Patient characteristics & No. (IQR) & $\%$ \\
\hline Age (years) & & \\
Median & $65(57.2-71.5)$ & \\
Gender & & 44.3 \\
Female & 39 & 55.7 \\
Male & 49 & \\
Tumor grade & & 53.4 \\
G2 & 47 & 46.6 \\
\hline G3 & 41 & \\
\hline
\end{tabular}

PDAC, pancreatic ductal adenocarcinoma.

compared using long-rank test (Mantel-Cox). All analyses were performed with SigmaPlot software.

More detailed information is available in the online supplemental material section.

\section{RESULTS \\ TERT is a versatile immune target in PDAC}

More than $95 \%$ of pancreatic cancer cases, regardless of the histological and molecular classification, exhibit enhanced TERT activity, when compared with normal tissues and benign pancreatic lesions. ${ }^{20}$ However, the relevance of TERT expression in patient prognosis has not yet been investigated. To address this issue, we enrolled a PDAC patient cohort $(n=88$, table 1$)$ based on the following inclusion criteria: resected pancreatic cancers and treatment-naïve patients. Immunohistochemistry (IHC) analysis revealed the presence of TERT antigen in most PDAC tissues, regardless of tumor stage. Notably, TERT was detected in $73 \%$ of the analyzed samples with either a highly intense motif $(++)$ or a positive framework (+) (figure 1A, B). Although TERT expression was not associated with a statistically significant overall survival, a fraction of long-term PDAC survivors displayed tumors

Table 2 Clinical features of the patients involved in the study of TME characterization

\begin{tabular}{lcc}
\hline Patient characteristics & No. (IQR) & $\%$ \\
\hline Age (years) & & \\
Median & $68(50-84)$ & \\
Gender & & \\
Female & 16 & 53.3 \\
Male & 14 & 46.7 \\
Tumor grade & & \\
G2 & 22 & 73.3 \\
G3 & 8 & 26.7 \\
\hline
\end{tabular}

TME, tumor microenvironment. expressing either moderate or high levels of the TERT antigen (online supplemental figure 1A).

We also tested TERT expression in the pancreatic tissues of $\mathrm{H}-2^{\mathrm{b}}$ inbred KPC mice, which spontaneously develop invasive PDAC, starting from precursor lesions identified as pancreatic intraepithelial neoplasia (PanIN).$^{521}$ We identified disease evolution from acinarto-ductal metaplasia (ADM) stage, characterized by lowgrade lesions with minimal cytological and architectural atypia, to PanIN\#1 stage marked by cytological abnormalities, PanIN\#2 lesions characterized by an intense degree of dysplasia, and eventually the PDAC disease stage (figure 1C). Notably, TERT antigen in mouse tissues increased according to tumor progression (figure 1C), and was mainly expressed in both mouse and human PDAC specimens (figure 1A and $\mathrm{C}$ ). In contrast, TERT immunostaining was present only in the Langerhans islets of both human and mouse normal pancreatic tissues.

Orthotopic transplantation of two different KPCderived cell lines, FC1242 (figure 1D) and FC1199 (online supplemental figure 1B), led to the development of TERT-expressing PDAC tumors that recapitulated KPC-associated cancer phases ${ }^{21}$ in a time window of 30 days. We confirmed increased in vitro TERT expression and enzymatic activity in these PDAC cells, as well as in several human PDAC cell lines (online supplemental figure 1C), compared with normal pancreas tissue (figure 1E). Finally, orthotopic injection of HF2 cells into immunodeficient mice generated TERT-positive tumors, suggesting that TERT expression was maintained during in vivo tumor growth (online supplemental figure 1D). Altogether, these data confirm that TERT is a preserved antigen during pancreatic cancer evolution and highlight its pertinence as immunotherapy target.

\section{In vivo efficacy of TERT-based immunotherapy as single treatment}

Toaddress the ability of PDAC cells to activate TERT-specific CTLs through correct antigen processing, we performed in vitro killing assays with either polyclonal mouse (m) TERT $_{198-205}$-specific $\mathrm{CD} 8^{+} \mathrm{T}$ lymphocytes or human (h) TERT $_{865-873}$-specific, TCR-engineered $\mathrm{T}$ lymphocytes that we previously characterized. ${ }^{10}{ }^{11}$ As expected, mouse TERT-specific CTLs specifically recognized PDAC cells in mouse $\mathrm{H}-2 \mathrm{~K}^{\mathrm{b}}$-restricted fashion, compared with the negative control, that is, $\mathrm{OVA}_{257-264}$-pulsed MBL2 cells (online supplemental figure 2A). Similarly, human (h) TERT ${ }_{865-}$ ${ }_{873}$-specific, human TCR-engineered $\mathrm{T}$ lymphocytes, recognized PDAC cells in HLA-A2-restricted fashion, compared with the negative control, that is, $\mathrm{HCV}_{1406-1415^{-}}$ pulsed, HLA A2 ${ }^{+}$, T2 cells, in terms of activation (online supplemental figure $2 \mathrm{~B}$, measured as IFN $\gamma$ release, white bar, right axis) and cytotoxic ability (online supplemental figure 2B, black bar, left axis). Notably, mouse and human anti-TERT lymphocytes killed PDAC cell lines to the same extent as TERT peptide-loaded controls MBL-2 cells and T2 cells, respectively. These data confirm that PDAC cells process and present TERT-derived peptides on class I 

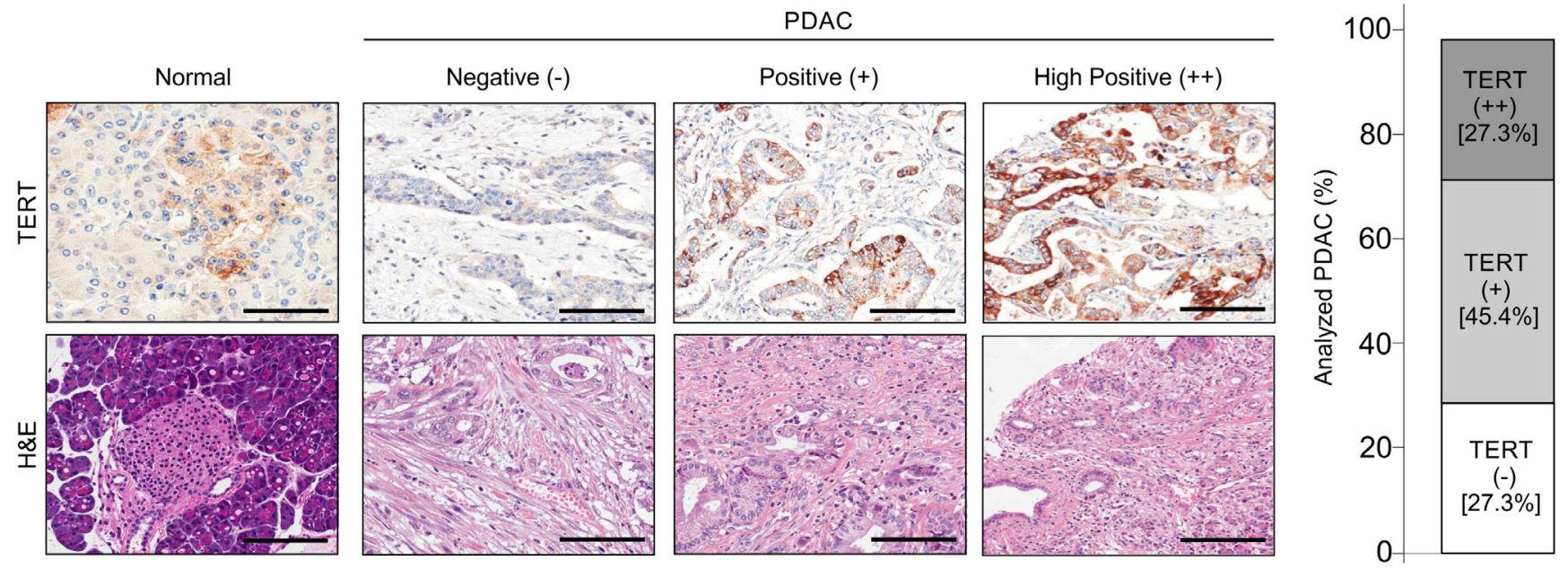

C
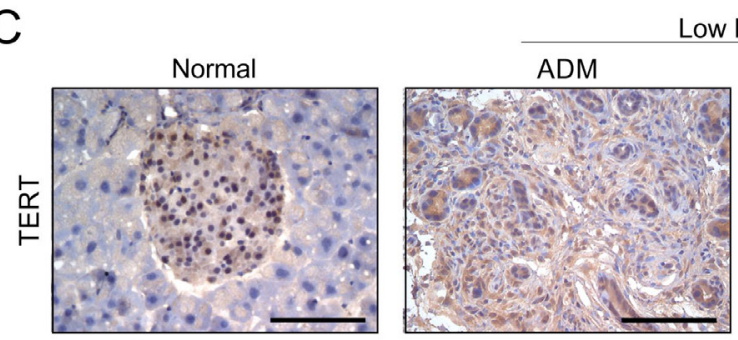

Low PanIN
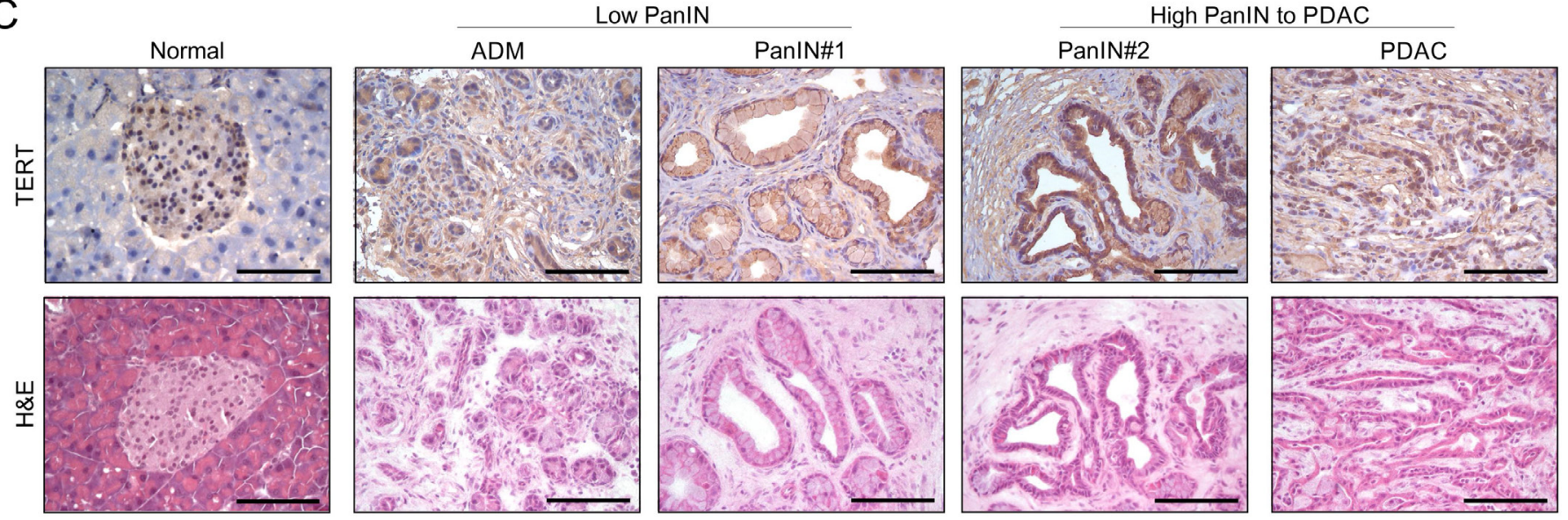

D
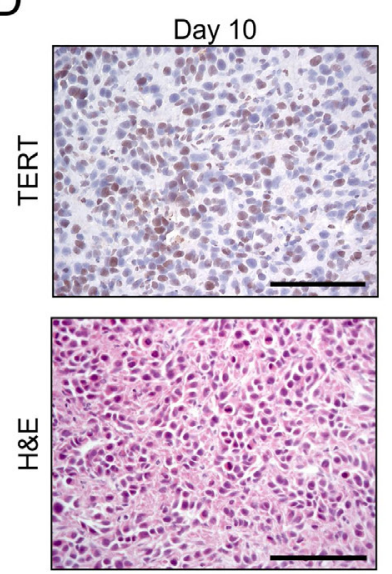
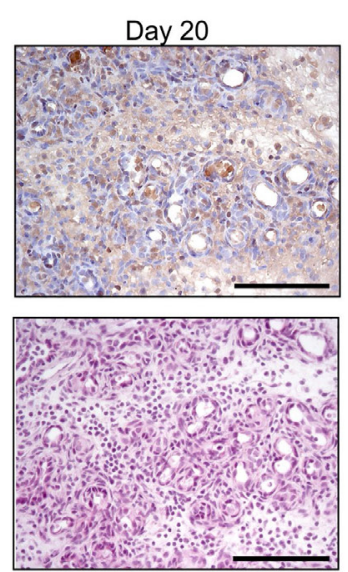
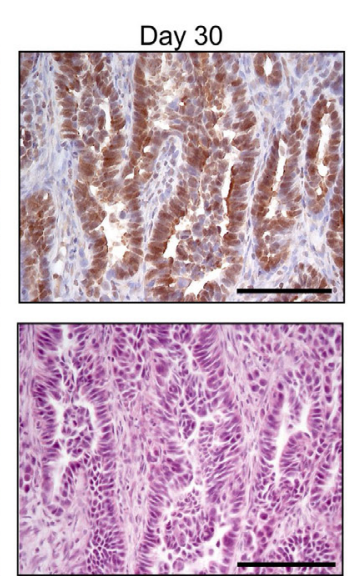

$E$

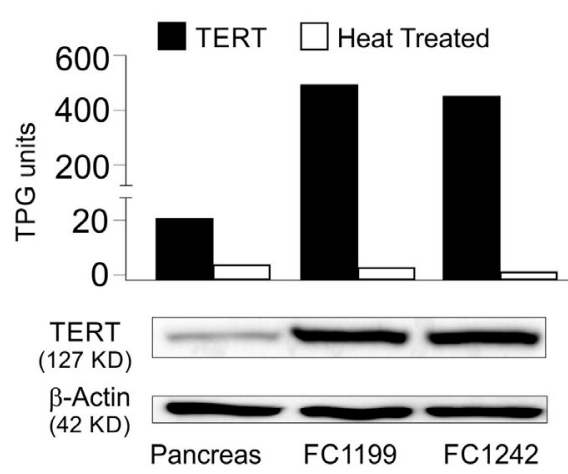

Figure 1 TERT expression in human and mouse PDAC tissues. (A) Representative H\&E staining and TERT IHC analysis in human normal pancreas and PDAC tissues (G2/G3 tumor grade). PDAC tissues were scored as negative (-), positive (+), highly positive (++) according to hTERT antigen. (B) Patients with pancreatic cancer were stratified according to TERT expression. (C) Representative TERT IHC analysis (top panel) and H\&E staining (bottom panel) in pancreas specimens isolated from KPC mice at different stages of neoplastic evolution, or in tumors isolated from C57BL/6 mice orthotopically injected with FC1242 cells (D) and sacrificed at different time points (10-30 days) from tumor challenge. Scale bar (A,C,D), $100 \mu \mathrm{m}$. (E) TERT activity and expression in normal pancreatic cells and KPC-derived cell lines by TRAP assay (top panel; untreated samples, black; heat-inactivated-negative control samples, white; TPG) and Western blot (bottom panel), respectively. Representative samples are shown. IHC, immunohistochemistry; PDAC, pancreatic ductal adenocarcinoma; TERT, telomerase; TPG, total product generated. 
major histocompatibility complex (MHC) proteins. To assess whether TERT-dependent immune killing resulted in cancer growth restriction in vivo, we treated s.c. FC1242 or FC1199 tumor bearing mice with two consecutive systemic infusions of either mTERT-specific (TERT-ACT) or control ovalbumin-specific (OVA-ACT) CTLs (online supplemental figure 2C). Tumor growth restriction was observed on TERT-ACT administration in both PDAC models, leading to a significant improvement in survival compared with the control (online supplemental figure 2D,E).

We then questioned whether immunotherapy could be effective when tumors grew in the original tissue environment. To answer this question, we engineered KPCderived cell lines with a firefly luciferase (Luc)-expressing lentivirus, generating FC1242-Luc and FC1199-Luc cells, to monitor tumor growth by bioluminescence with in vivo imaging. After proving that the transduced cells were recognized to the same extent by mouse TERT-specific CTLs (online supplemental figure 2A) and exhibited similar tumor growth as their parental counterparts (online supplemental figure $2 \mathrm{~F}$ ), we orthotopically transplanted Luc-expressing PDAC cells into immunocompetent mice to evaluate the therapeutic effectiveness of TERT-based ACT (online supplemental figure 2G). In this experimental setting, TERT-based immunotherapy partially restricted both FC1242-Luc (figure 2A) and FC1199-Luc (figure 2B) tumor progression, and improved mouse survival. Several factors may be responsible for limited immunotherapy efficacy. T lymphocyte-intrinsic factors linked to the $\mathrm{T}$ effector memory phenotype of CTLs employed in our ACT protocol that express also exhaustion markers (ie, programmed death (PD1), online supplemental figure $2 \mathrm{H}$ ) strongly affect the in vivo antitumor efficacy through their limited proliferation potential. ${ }^{22} \mathrm{~T}$ lymphocyte-extrinsic factors are mainly due to tumor features that may hinder $\mathrm{T}$ lymphocyte infiltration by multiple strategies, avoiding $\mathrm{T}$ lymphocytemediated tumor immune restriction. ${ }^{23}$ In accordance with this hypothesis, we identified few adoptively infused TERT-specific CTLs (CD45.2 ${ }^{+}$cells) in orthotopic tumors isolated from congenic (CD45.1) mice (figure 2C). Therefore, vessels and ECM modifications as well as the presence of multiple inhibitory soluble factors in the $\mathrm{TME}^{4}$ limit but do not completely abrogate T lymphocyte trafficking inside pancreatic tumors.

To extend these results to mice that spontaneously develop PDAC, we treated a cohort of KPC mice with either TERT-specific or OVA-specific $\mathrm{T}$ lymphocytes (online supplemental figure 2I). A significant TERT-dependent tumor control was achieved in KPC mice (figure 2D), confirming the potential of adoptive immunotherapy in PDAC. To address whether TERT-based immunotherapy could be suitable in a preclinical human PDAC setting, we orthotopically engrafted immunodeficient NOG mice with HLA-A2 ${ }^{+}$PDAC, Luc-expressing cells (HF2-Luc cell line) and treated them with repeated injections of either hTERT $_{865-873}$-specific (TERT-ACT) or $\mathrm{HCV}_{1406-1415}$-specific,
TCR-engineered $\mathrm{T}$ lymphocytes (HCV-ACT, Ctrl) followed by in vivo detection of bioluminescence (online supplemental figure 2G). TERT-based immunotherapy significantly improved tumor control and mouse survival (figure 2E). Collectively, our data reveal the ability of TERT-specific CTLs to reach the TME, thereby triggering an immune-based tumor control.

\section{Pancreatic cancers establish an immunosuppressive barrier that limits the T lymphocyte-mediated response}

Considering the limited impact of TERT-based ACT on survival, we investigated the TME to identify factors that might restrain $\mathrm{T}$ lymphocyte homing to tumor core and cytotoxic activity. TME in PDAC comprises immunosuppressive myeloid cells, such as MDSCs and TAMs. ${ }^{24}{ }^{25}$ We performed a deep analysis of the tumor immune microenvironment evolution at different time-points: day 10, day 20, and day 30 post-tumor challenge. Both FC1242derived and FC1199-derived orthotopic tumors gradually shaped the immune contexture in consecutive developmental stages, resulting in progressive ECM deposition, $\mathrm{T}$ lymphocyte contraction, and increased myeloid cell infiltration (figure 3A, B). Indeed, we found an inverse correlation between the percentage of $\mathrm{T}$ lymphocytes and the frequency of either tumor-infiltrating myeloid cells $\left(\mathrm{CD} 11 \mathrm{~b}^{+}\right.$cells $)$or MDSCs in both experimental models using flow cytometry (figure 3C, D). Furthermore, using multiparametric flow cytometric staining (online supplemental figure 3A), we characterized different myeloid cell subsets: TAM-MHCII ${ }^{\text {hi }}\left(\mathrm{CD}^{1} 1 \mathrm{~b}^{+}\right.$Ly6G Ly6C $^{-}$

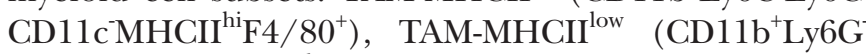
Ly6C CD11c-MHCII ${ }^{\text {low }} \mathrm{F} 4 / 80^{+}$), dendritic cells

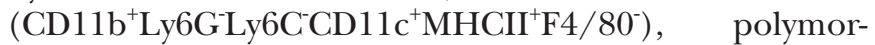

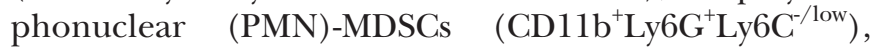

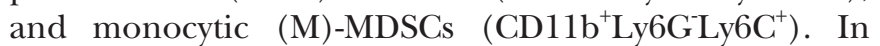
the FC1242 orthotopic model, a shift in the immune composition of the tumor-infiltrating $\mathrm{CD}_{11} \mathrm{~b}^{+}$cells could be mainly ascribed to a consistent accumulation of PMNMDSCs, which reached $16 \%$ at day 30 from $6.8 \%$ at the first time-point $(p=0.012)$, associated with a progressive increase in M-MDSC frequency from 3\% to 9\% $(\mathrm{p}=0.034)$ (figure 3E). Notably, we also identified a switch between TAM-MHCII ${ }^{\text {hi }}$, which declined from $49.7 \%$ to $13.7 \%(\mathrm{p}<0.001)$ and TAM-MHCII ${ }^{\text {low }}$, which consistently increased to $30 \%$ of the total PDAC-associated myeloid cells at late time-points of disease progression, after starting from an initial frequency of $5.7 \% \quad(p=0.001)$ (figure 3E). The same immunological landscape was observed in FC1199 tumors (PMN-MDSCs increasing from $14 \%$ to $32 \% \mathrm{p}=0.01$; TAM-MHCII ${ }^{\text {low }}$ ranging from $16 \%$ to $32 \%, \mathrm{p}=0.002$, TAM-MHCII ${ }^{\text {hi }}$ decreasing from $23 \%$ to $5 \% \mathrm{p}=0.002$ ) confirming a constant immunosuppressive milieu development during cancer progression characterized by MDSCs and TAM-MHCII ${ }^{\text {low }}$ accumulation (figure $3 \mathrm{~F}$ ).

To establish how tumors could promote MDSC and TAM infiltration, we explored the cytokine milieu produced by KPC-derived cell lines ex vivo and in vitro. We uncovered 
A

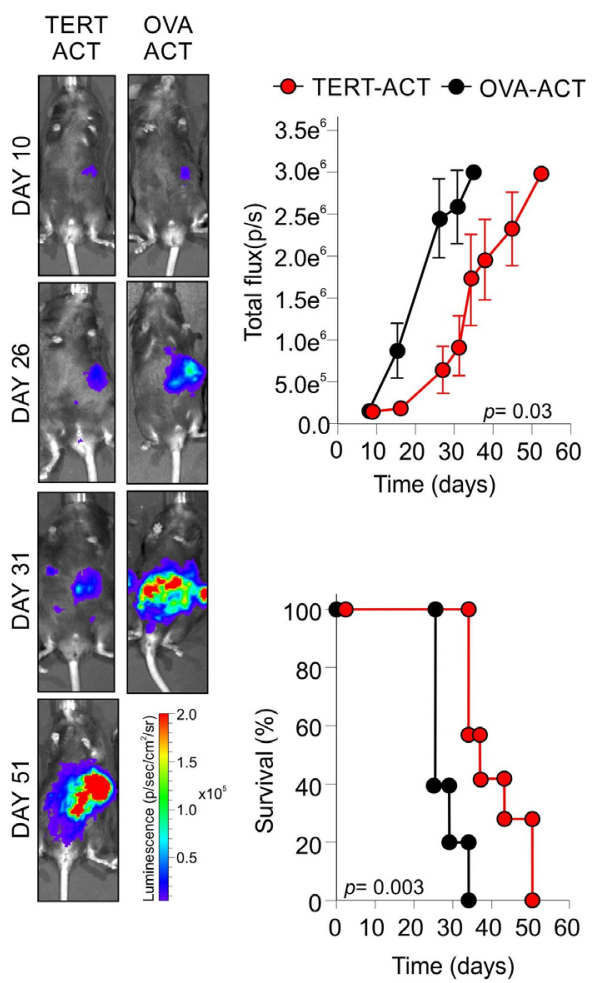

B

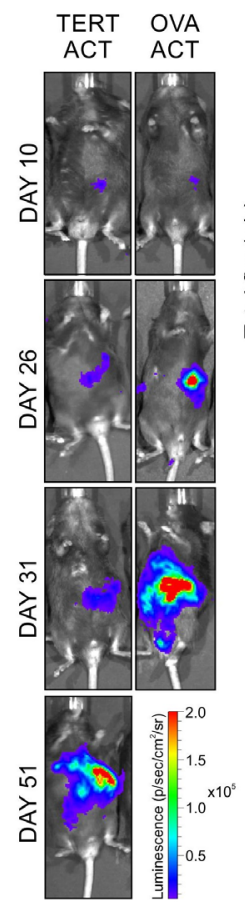

C
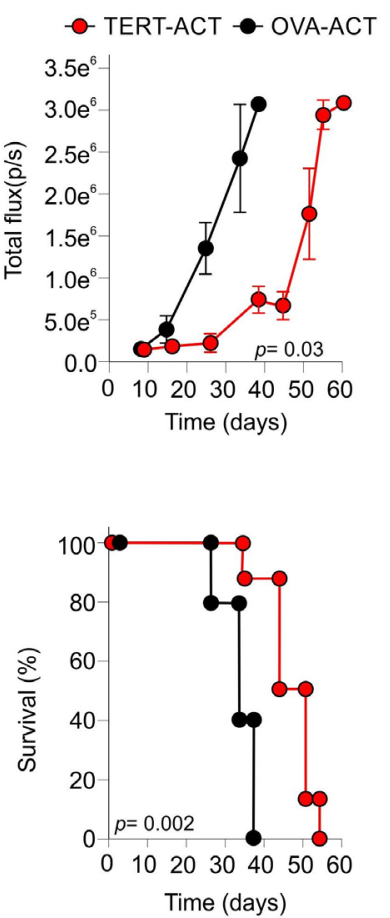

c
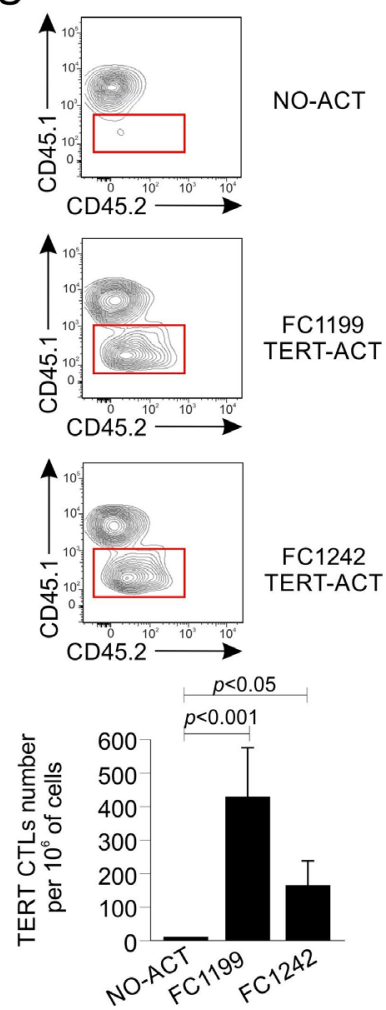

D

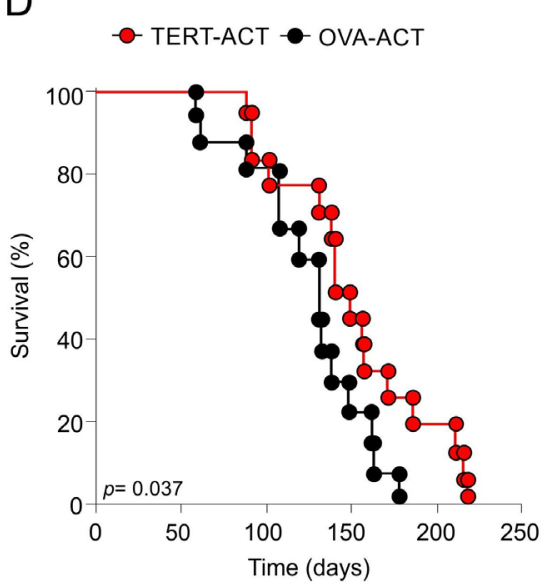

$E$

Figure 2 TERT-based ACT restrains PDAC progression. (A,B) C57BI/6 mice were orthotopically challenged with mouse KPC-derived FC1242-Luc (A) or FC1199-Luc (B) cells. Mice were randomized in two groups which received mTERT-specific (treatment group, $n=10$ ) or OVA-specific (control group $n=10$ ) mouse CTLs ACT. Therapeutic efficacy was evaluated over time in terms of tumor progression (top panel) and survival (bottom panel). (C) Tracking of adoptively transferred T lymphocytes in PDAC specimens. Congenic immunocompetent CD45.1 mice were orthotopically challenged with mouse KPC-derived FC1242 or FC1199 cells and subjected to CD45.2 $2^{+}$mTERT-specific mouse CTLs ACT or left untreated. Tumor-infiltrating T lymphocytes $\left(\mathrm{CD}^{+} \mathrm{CD}^{+}\right.$cells) were stained with both $\mathrm{CD} 45.1$ (endogeneous lymphocytes) and CD45.2 (transferred lymphocytes) antibodies. (D) KPC mice were enrolled in two groups receiving mTERT-specific (treatment group, $n=20$ ) or OVA-specific (control group $\mathrm{n}=20$ ) mouse CTLs ACT. Therapeutic efficacy was evaluated over time by Kaplan-Meier curves for OS. (E) Immunodeficient NOG mice were orthotopically challenged with human PDAC HLA-A2 ${ }^{+}$HF2_Luc cell line. Mice were randomized in two groups which received, respectively, hTERT-specific (treatment group) or HCV-specific (control group) engineered human T lymphocytes. Tumor growth was monitored by in vivo imaging (left panel). Therapeutic efficacy was evaluated over time in terms of tumor progression (middle panel) and survival (right panel). Tumor growth was evaluated by in vivo imaging (IVIS-Perkin Elmer: A,B,E; Vevo ultrasound imaging: D): a representative image for each analyzed time point was reported (A,B,E). Data are reported as mean $\pm S E$ of a representative experiment of two $(A, B, E)$ and three $(C)$ independent replicates. Statistical analysis was performed using one-wayANOVA (A,B,E), Mantel-Haenszel (long-rank) test (A, B, D, E), two-tailed Student's $t$-test (C). ACT, adoptive cell therapy; ANOVA, analysis of variance; CTL, cytotoxic T lymphocyte; OS, overall survival; PDAC, pancreatic ductal adenocarcinoma. 
A

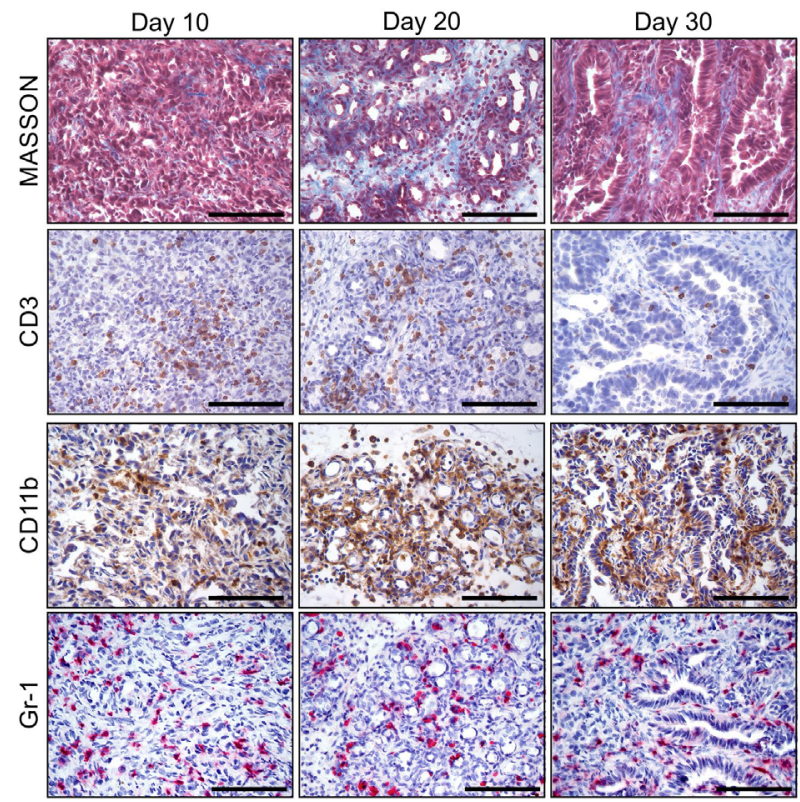

C
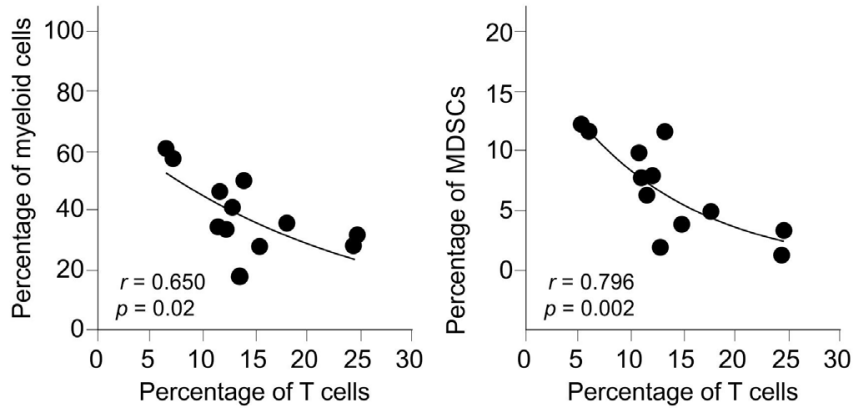

$E$

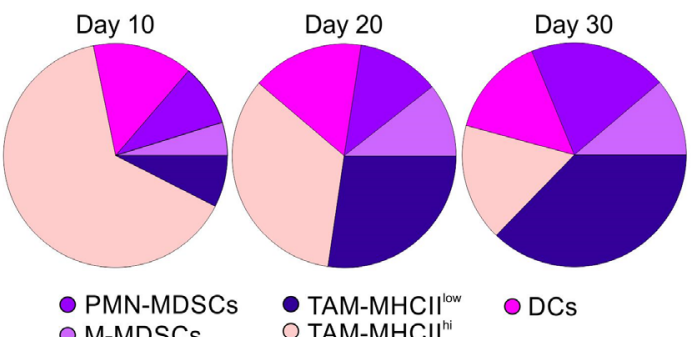

B

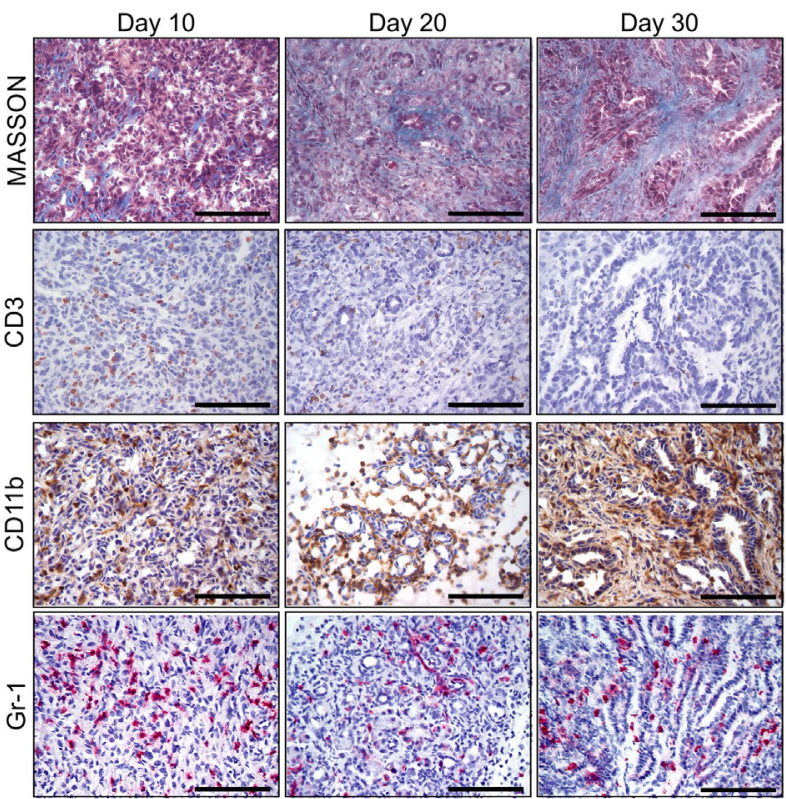

D
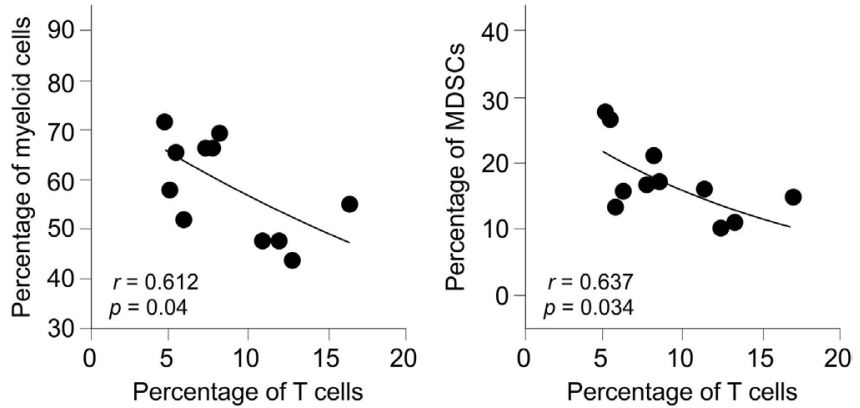

$\mathrm{F}$

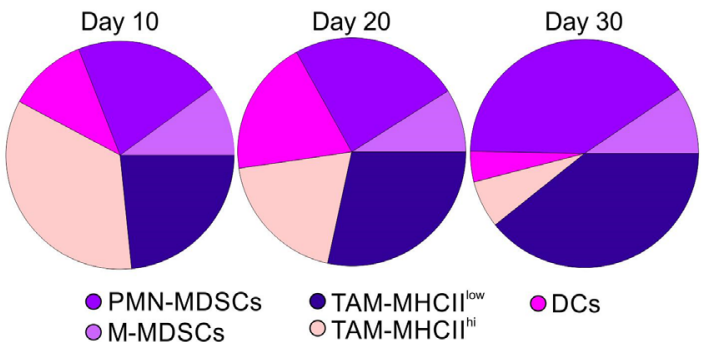

Figure 3 Immunosuppressive tumor-infiltrating myeloid cells limit T lymphocytes trafficking. (A,B) C57BI/6 mice were orthotopically challenged with mouse KPC-derived FC1242 (A) or FC1199 (B) cells. Mice were sacrificed at different time points from tumor challenge. Representative Masson Trichrome and IHC staining for CD3, CD11b and Gr-1 tumor specimens at different time point of PDAC progression. Scale bar, $100 \mu \mathrm{m}$. (C,D) Tumors were enzymatically digested to isolate cell suspensions and processed for immune phenotype analysis using the following markers: CD45, CD11b, Ly6C, Ly6G, CD11c, MHCII, CD3, CD4, and CD8. Spearman's rank correlation of cell frequency between tumor-infiltrating myeloid cells (CD11 b cells) or MDSCs (CD11b Ly6C $^{+}$Ly6G $^{-}$M-MSDCs plus CD11 b ${ }^{+}$Ly6C $^{-/ l o w}$ Ly6G $^{+}$PMN-MDSCs) and T lymphocytes (CD3 ${ }^{+}$cells) during FC1242 (C) or FC1199 (D) tumor evolution. Each plot refers to a pool of three mice. (E,F) Characterization of tumor infiltrating myeloid cells subsets during $\mathrm{FC1242}(\mathrm{E})$ or $\mathrm{FC} 1199(\mathrm{~F})$ tumor progression, $n=5$ mice/time point. Myeloid cells were divided into: M-MDSC ( $\mathrm{Ly} 6 \mathrm{C}^{+}$Ly6G- cells), PMN-MDSC ( Ly6C $^{-/ 10 w} \mathrm{Ly}^{-} \mathrm{G}^{+}$cells) or macrophages/DCs characterized as: Ly6C-

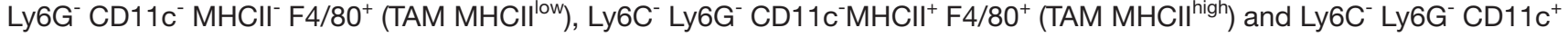
$\mathrm{MHCII}^{+}(\mathrm{DCs})$, respectively. On the contrary, T lymphocytes were characterized as $\mathrm{CTL}\left(\mathrm{CD}^{+}{ }^{+} \mathrm{CD} 8^{+}\right.$cells) or $\mathrm{T}_{H}\left(\mathrm{CD}^{+}{ }^{+} \mathrm{CD} 4^{+}\right.$cells) lymphocytes. Within the CD11 $b^{+}$cell subsets, PMN-MDSCs $(p=0.01(E-F))$ and TAM-MHCIlow $(p<0.001(E), p=0.001(F))$ were those dramatically increasing according to tumor progression in spite of TAM-MHCII ${ }^{\text {hi }}(p=0.001(E), p=0.02(F))$. CTL, cytotoxic T lymphocyte; MDSC, myeloid-derived suppressor cell; PDAC, pancreatic ductal adenocarcinoma. 
a peculiar inflammatory signature, characterized by high levels of granulocyte monocyte colony-stimulating factor, interleukin (IL)-6, IL-10, monocyte chemoattractant protein-1 (also known as CCL2), and vascular endothelial growth factor (online supplemental figure 3B,C). These cytokines, which are crucially involved in MDSC expansion, recruitment, and differentiation, as well as in TAM polarization toward M2 protumoral functions, ${ }^{26}$ are produced mainly by tumor cells (online supplemental figure 3D); many of these proteins are positively controlled by KRAS oncogenic activation. ${ }^{27}$ Nonetheless, we cannot exclude the possibility that other components of the TME (eg, CAFs) could also be actively involved in their release in vivo.

Finally, we proved that PDAC-infiltrating myeloid cells were endowed with highly immunosuppressive features toward T lymphocytes. Purified CD $11 \mathrm{~b}^{+}$cells from both FC1242 and FC1199 tumors significantly constrained both the in vitro killing ability and the proliferation of antigen-specific CTLs (online supplemental figure 3E,F). Additionally, we evaluated the frequency (online supplemental figure 3G) and immunosuppressive function (online supplemental figure $3 \mathrm{H}$ ) of circulating $\mathrm{CD}_{11 \mathrm{~b}^{+}}$ cells isolated from the spleen of both FC1242 and FC1199 tumor-bearing mice, confirming their ability to affect $\mathrm{T}$ lymphocyte response, even at a lower level compared with the tumor-infiltrating counterpart.

To address whether this immunosuppressive landscape was also established during autochthonous tumor evolution, we analyzed KPC mice. Consistently, the immune composition of KPC-derived tumors revealed a considerable accumulation of $\mathrm{CD}_{1} 1 \mathrm{~b}^{+}$cells during PDAC progression. In contrast, $\mathrm{T}$ lymphocytes $\left(\mathrm{CD}^{+}\right.$cells) were detected mainly in the early phases of PDAC progression (online supplemental figure 4A). Indeed, the frequencies of tumor-infiltrating $\mathrm{T}$ lymphocytes $\left(\mathrm{CD}^{+}\right.$cells), CTLs $\left(\mathrm{CD}^{+} \mathrm{CD}^{+}\right.$cells $)$, and helper $\mathrm{T}$ lymphocytes $\left(\mathrm{CD}^{+} \mathrm{CD}^{+}\right.$ cells) were inversely correlated with the frequency of myeloid cells $\left(\mathrm{CD} 11 \mathrm{~b}^{+}\right.$cells), MDSCs (CD11b ${ }^{+} \mathrm{Ly}-$

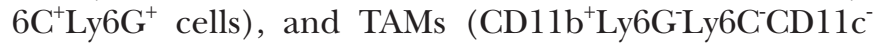
$\mathrm{MHCII}^{\mathrm{low}} \mathrm{F} 4 / 80^{+}$) (online supplemental figure $4 \mathrm{~B}$ ).

As NOS2 and ARG1 are among the main players of MDSC-mediated and TAM-mediated immune suppression, we evaluated their expression in PDAC TME. WB analysis identified NOS2 and ARG1 only in FC1242derived and FC1199-derived tumors and tumorinfiltrating $\mathrm{CD}_{11 \mathrm{~b}^{+}}$cells regardless of tumor stage (figure 4A, B); CD11 b cells were among the sources of both enzymes. ARG1 and NOS2 metabolize L-arginine to produce urea $+\mathrm{L}$-ornithine and $\mathrm{NO}+\mathrm{L}$-citrulline, respectively. In the TME, catabolism of L-arginine may generate RNS-dependent hurdles that protect cancer cells from immune attack, as shown in different transplantable tumor models. ${ }^{19}$ Although ARG1 is widely considered a hallmark of immunosuppressive myeloid cells, the role of NOS2 in the regulation of antitumor immunity is more context-dependent, since it can both restrict or support $\mathrm{T}$ lymphocyte immunity according to the immune cell subset that expresses it and the presence of a tumorspecific T cell response. ${ }^{1728}$ However, in the context of low immunogenic cancers, such as PDAC, NOS2 activity correlates with increased tumor invasiveness and resistance to therapy. ${ }^{29}$

We proceeded to determine whether the establishment of N-Ty-based chemical barrier could affect T lymphocyte infiltration in the PDAC TME. We identified an inverse distribution of $\mathrm{T}$ lymphocytes and N-Ty-positive tumor areas in both the FC1242 and FC1199 cancer specimens: a progressive exclusion of CTLs from the PDAC tumor core was indeed associated with the establishment of a marked N-Ty-based shield (figure 4C, D). Furthermore, a consistently increased N-Ty pattern in KPC mice from PanIN phases to locally invasive tumors (figure 4E) was confirmed, together with an opposite distribution of $\mathrm{CD}^{+} \mathrm{T}$ lymphocytes and N-Ty-positive tumor regions. Notably, T lymphocytes were detected only in the early phases of PDAC development (PanIN\#1), while they almost disappeared in the late tumor stage (PDAC). We then evaluated whether a similar landscape could be identified in human PDAC (table 2). Despite the interpatient and intrapatient immune heterogeneity that characterizes pancreatic cancer, ${ }^{1430}$ an inverse correlation in the spatial distribution of myeloid cells, detected as $\mathrm{CD} 68^{+}$ macrophages or, more often, $\mathrm{CD}_{1} 5^{+}$neutrophils and $\mathrm{CD}^{+} \mathrm{T}$ lymphocytes, was evident in all examined specimens $(n=30)$. While myeloid cells were identified both in the peripheral and in the central zone of invasive tumors, $\mathrm{T}$ lymphocytes were mostly found around the peripheral tumor glands. In addition, we also identified N-Ty staining on infiltrating immune cells or on tumor glands, close to the zones of $\mathrm{CD}^{+} \mathrm{T}$ cell infiltration, in about $25 \%$ of analyzed specimens (figure $4 \mathrm{~F}$ ), mirroring the preclinical PDAC setting. Notably, N-Ty deposition was evident in both tumor and stromal tissue (figure $4 \mathrm{C}-\mathrm{F}$ and online supplemental figure $5 \mathrm{~A}$ ), whereas in normal pancreatic samples it could be identified only in endocrine islets.

Together, these data suggest an immune evasive pattern that is shared between human and mouse PDAC, in which the accumulation of immunosuppressive myeloid cells inside the TME promotes the establishment of an N-Tydependent $\mathrm{T}$ lymphocyte trap by generating RNS, which impairs the T lymphocyte accrual to the tumor core.

\section{RNS-targeting contributes to enhanced cancer immunotherapy in PDAC}

Despite encouraging results of approaches targeting MDSC/TAM accumulation, recruitment, or function, their clinical benefits still need to be confirmed in the context of PDAC. ${ }^{31}{ }^{32}$ An array of scavengers for peroxynitrite-derived radicals and metalloporphyrinic antioxidants have been developed to block RNS generation; ${ }^{33}$ however, most are not bioavailable and may thus have limited therapeutic potential. Recently, we designed a new compound, AT38 (cas. 46843548), that can affect RNS generation. ${ }^{19}$ We sought to investigate 
A

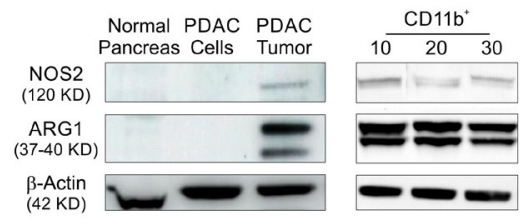

C

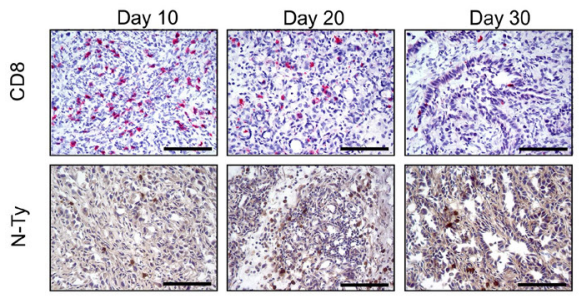

D

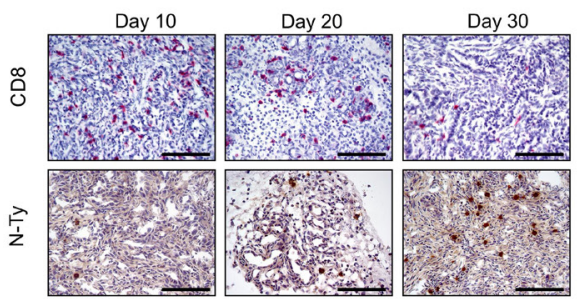

E

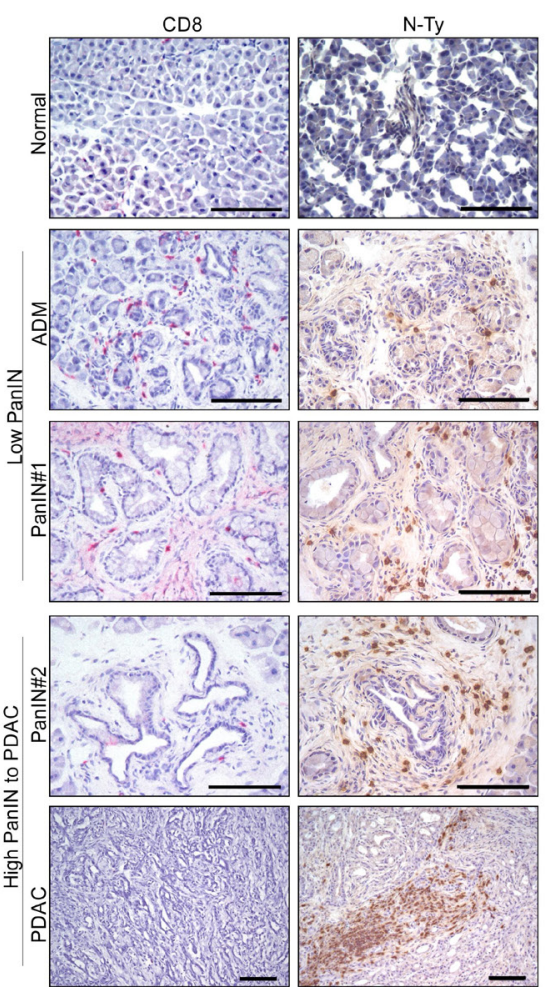

B

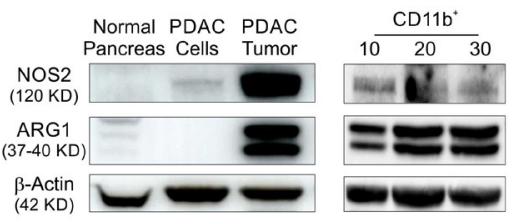

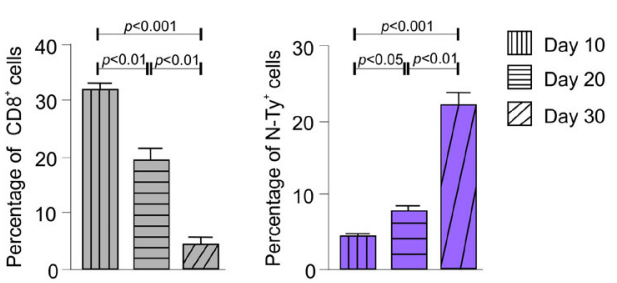
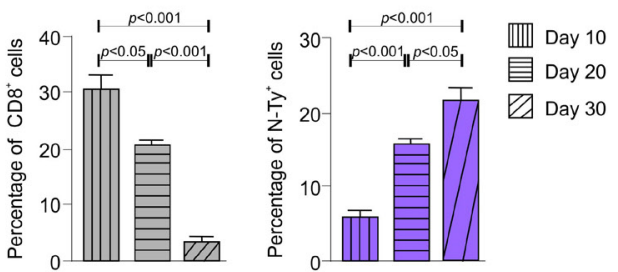

$\mathrm{F}$

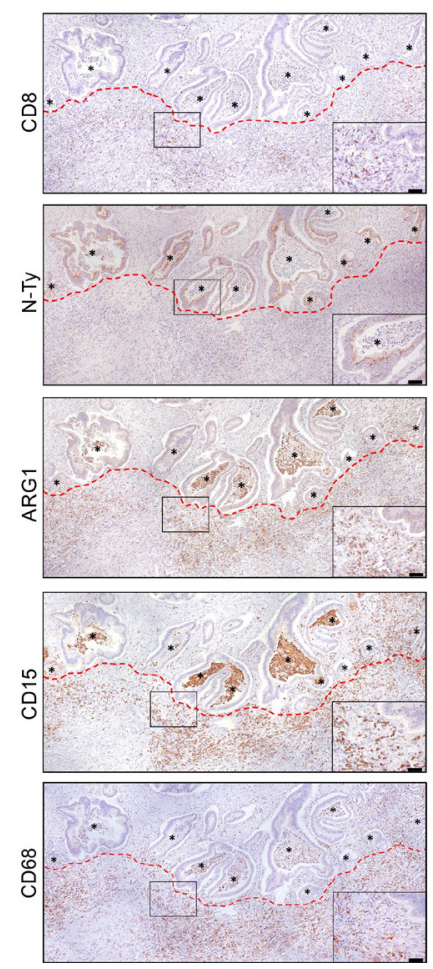

Figure 4 NTy-based deposition is a hallmark of immunotherapy resistance in PDAC. (A,B) ARG1 and NOS2 expression in normal pancreas, PDAC cells, PDAC tumor, tumors-isolated $\mathrm{CD}_{11} \mathrm{~b}^{+}$cells at different stages of tumor progression (day 10 , 20, 30 since tumor challenge) in FC1242 (A) and FC1199 (B) models, respectively. (C-E) Either, respectively, FC1242 (C) or FC1199 (D) tumor samples or KPC-derived pancreas specimens (E) at different stages of tumor progression, were analyzed for the presence of TILs and of RNS by IHC staining for CD8 and N-Ty. Non-contiguous ROIs with dimensions of $157 \times 157$ pixels were analyzed for both N-Ty or CD8 staining and applied to serial sections. The percentages of immunoreactive areas for both markers were calculated for each ROI. Data are expressed as means $\pm S D$ of $R O I(n=20)$. Statistical analysis was performed by a one-way ANOVA, followed by Tukey's test. (F) Representative IHC staining for CD8, N-Ty, ARG1, CD15, CD68, in human PDAC to evaluate the presence and localization of CTLs, RNS, ARG1, neutrophils and macrophages, respectively. Tumor glands are indicated with '*'. Scale bar, $100 \mu \mathrm{m}(\mathrm{C}-\mathrm{F})$. ANOVA, analysis of variance; CTL, cytotoxic T lymphocyte; IHC, immunohistochemistry; PDAC, pancreatic ductal adenocarcinoma; ROI, regions of interest. 
whether AT38 modulates nitrosative stress in the PDAC microenvironment.

First, we addressed the ability of AT38 to abrogate NOS2 and ARG1 expression in both in vitro differentiated bone marrow-derived macrophages (figure 5A, B) and MDSCs (figure 5C). The transcriptional inhibition of the two genes restored the in vitro proliferation of antigen-specific CTLs (figure 5D, E). Moreover, AT38 treatment metabolically reprogrammed both M1-polarized and M2-polarized macrophages by blocking arginine consumption and limiting the production of ornithine (figure $5 \mathrm{~F}$ ). We also revealed an AT38-dependent modulation of energetic metabolism, restricted to M1-polarized macrophages by Seahorse extracellular flux analyser (online supplemental figure 6A-C). AT38 treatment promoted a significant increase of basal, maximal respiration and ATP production rate, suggesting an increased OXPHOS capacity in AT38 treated compared with untreated M1 macrophages (figure 5G). Finally, we assessed AT38 impact on rewiring T lymphocyte energetic metabolism (online supplemental figure 6D). AT38 treatment in activated CTLs improved respiratory parameters such as basal, maximal and ATPlinked respiration (figure 5H), highlighting an increased mitochondrial bioenergetics in treated cells. Moreover, AT38 administration enhanced the energetic metabolism highlighted by the increased oxygen consumption rate (OCR) and extracellular acidification rate (ECAR), indicative of oxidative and glycolytic metabolism, respectively (figure 5I).

To gain further insights into the reprogramming cues induced on AT38 treatment, we supplied a daily administration of AT38 (for a week) to PDAC tumor-bearing mice that were orthotopically engrafted with FC1242 cells. We then performed single-cell RNA sequencing (scRNA-seq) analysis of the PDAC-infiltrating immune cells. Postquality control, we obtained 8454 and 7156 cells in the control (CTRL) and AT38 samples, respectively. Following reference-based classification and manual inspection (online supplemental figure 7A), the identified immune cells were visualized using uniform manifold approximation and projection (UMAP), followed by assessment of their proportions (figure 6A). In AT38-treated mice, we observed a decrease in the PMN-MDSC proportion (37\% CTRL; 28\% AT38) and an increase in T/NKT lymphocytes (20\% CTRL; 29\% AT38), whereas no relevant changes were identified in M-MDSCs and TAMs (figure 6A). Although we did not observe any imbalance in macrophage and monocyte composition, we found that AT38 treatment re-educates all the myeloid compartments to support antitumor immunity. Gene set enrichment analysis (GSEA) underscored an upregulation of interferon $\alpha / \gamma$ response pathways ( $C d 74$ and $I s g 15$, figure $6 \mathrm{~B}$ ), and a downregulation of pathways associated with tumor immune tolerance. Indeed, genes involved in immunosuppression (Cebpb, Arg1, and Spp1), angiogenesis (Spp1 and S100a4), as well as ECM deposition and support of tumor epithelial-to-mesenchymal transition (EMT) (Spp1, Fn1, Ecm 1, Vim, and Thbs1) were downmodulated in TAMs (figure 6C). AT38 equally induced the activation of interferon $\alpha / \gamma$ response in M-MDSCs (B2m, Isg15, Bst2, Ifitm3, and Zbp1) and PMN-MDSCs (Stat1, Psmb8, Psmb9, Tapbp, $B 2 m$, and $I s g 15$ ) (online supplemental figure 7B-E). GSEA also revealed the downregulation of genes involved in ECM deposition, support of tumor EMT ( $F n 1$ and Ecm1) and Mtorc1 signaling (Aldoa1) in M-MDSCs (online supplemental figure 7B) and upregulation of genes involved in oxidative phosphorylation ( $M d h 2$ and Atp5k) in PMN-MDSCs (online supplemental figure 7D).

We further validated the impact of AT38 treatment in reprogramming of PDAC TME in mice bearing FC1242 orthotopic tumors. We detected a remarkable reduction in the N-Ty-positive tumor area in AT38-treated mice (figure 6D), together with decreased ECM deposition and lower expression of ARG1 and NOS2 (figure 6D, E). As expected, AT38 administration promoted an immune reorganization of the TME, characterized by an intense accumulation of $\mathrm{T}$ lymphocytes and a contraction in tumor-infiltrating myeloid cells (figure $6 \mathrm{~F}$, online supplemental figure 8A). More precisely, we identified an increased frequency of CTLs $(4.7 \%$ vs $6.6 \%, \mathrm{p}=0.05)$ and $\mathrm{CD}^{+} \mathrm{T}$ lymphocytes (6.5\% vs $\left.9.7 \%, \mathrm{p}=0.04\right)$, with a concomitant reduction in total MDSCs $(15.8 \%$ vs $11.7 \%$, $\mathrm{p}=0.035$ ) in tumors isolated from treated mice (figure $6 \mathrm{~F}$ ). Furthermore, we proved that tumor-infiltrating CD11b ${ }^{+}$ cells isolated from AT38-treated mice partially lost their immunosuppressive properties (figure 6G). We substantiated these findings in a second KPC-derived tumor model. Characterization of the TME of the AT38-treated FC1199bearing mice revealed a similar immune rearrangement, marked by an increased infiltration of lymphocytes at the expenses of the immunosuppressive myeloid cells (online supplemental figure 8B-E). Moreover, treatment with AT38 reduced ECM deposition as well as ARG1 and NOS2 expression (online supplemental figure 8C, D). Accordingly, tumor-infiltrating myeloid cells exhibited defective inhibition of $\mathrm{T}$ lymphocyte proliferation (online supplemental figure $8 \mathrm{~F}$ ).

Finally, we addressed whether TME manipulation by AT38 could improve the therapeutic effectiveness of TERT-based ACT in PDAC. Therefore, tumor-bearing mice orthotopically engrafted with FC1242 cells received either single-arm treatment (AT38 alone or TERT-ACT alone) or combined therapy (online supplemental figure 9A). TME reprograming by AT38 improved the therapeutic impact of adoptively transferred, TERT-specific CTLs, leading to significant control of tumor growth (figure 7A) and significant survival benefit for the treated PDAC-bearing mice (figure 7A). More importantly, we verified the efficacy of this combined immunotherapeutic approach for the treatment of KPC mice (online supplemental figure 9B). Mice treated with AT38 plus TERT-ACT displayed substantially restricted tumor growth compared with untreated or single-arm treated mice, thereby suggesting that the treatment promotes some objective tumor regression (figure 7B) and a 
A

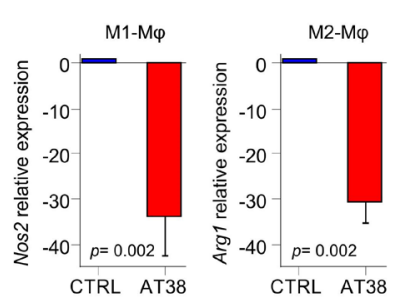

D

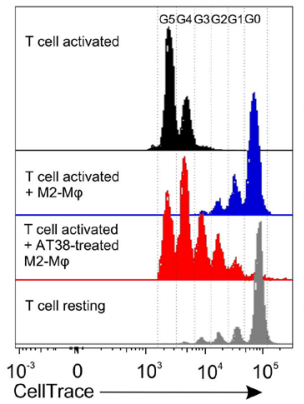

B

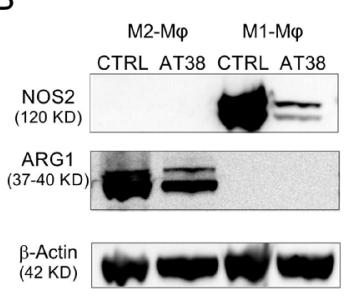

C

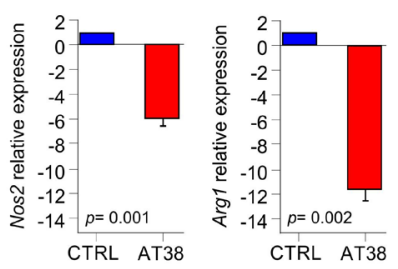

E
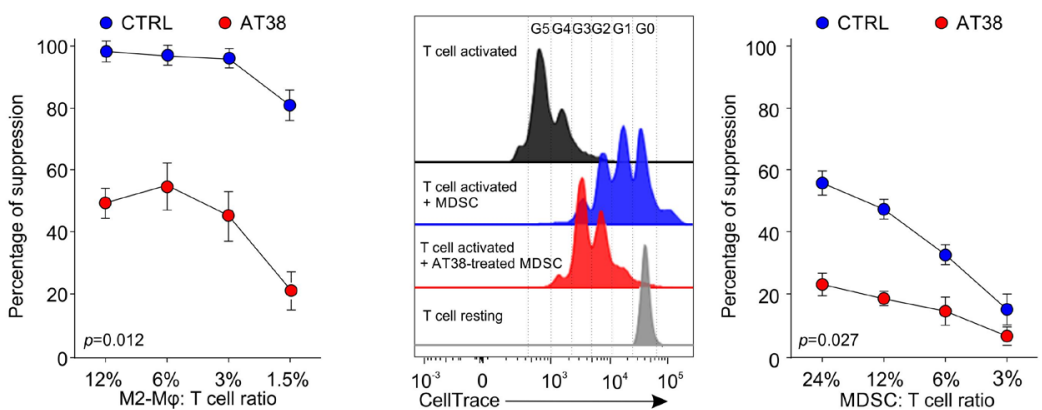

$\mathrm{F}$

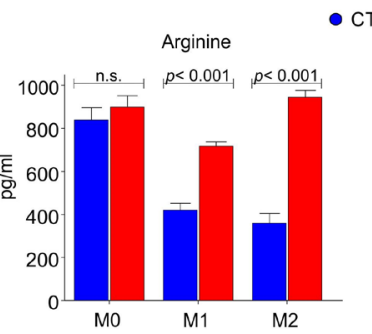

G

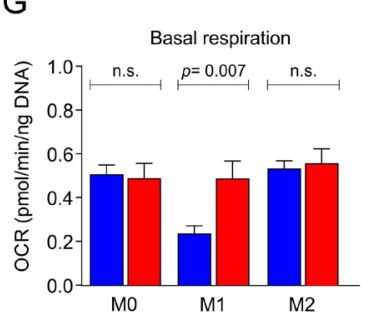

- ctrl
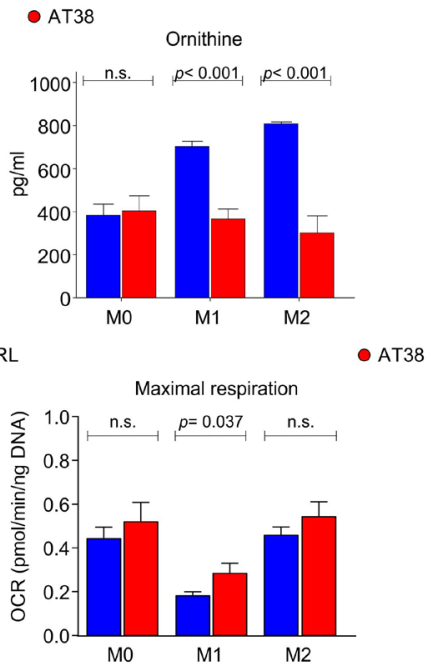

$\mathrm{H}$

- CTRL

- AT38

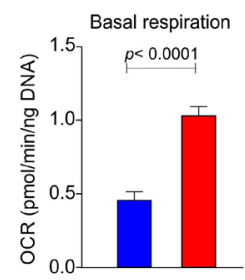

Maximal respiration
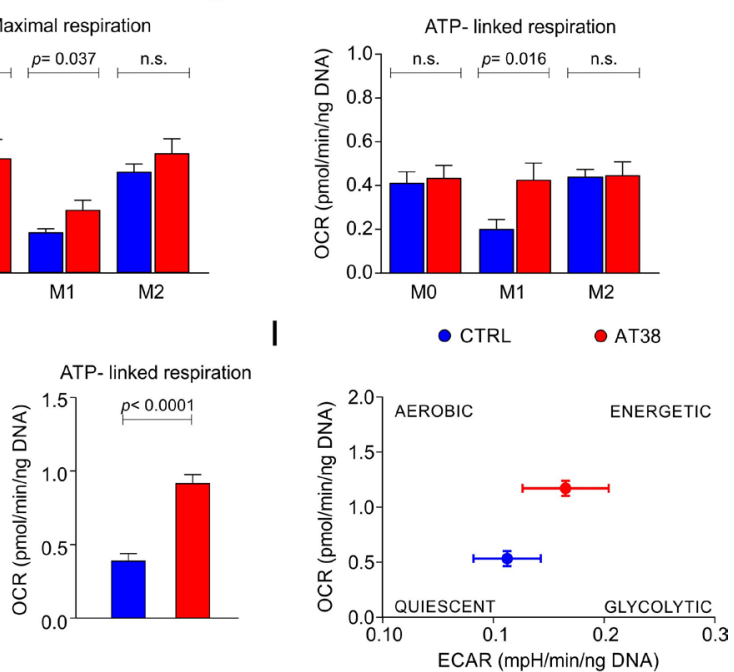

Figure 5 AT38 reprograms immune suppressive myeloid cells in vitro. (A,B) In vitro BM-derived M1 and M2-polarized macrophages (M $\mathrm{\phi})$ were treated for 24 hours with AT38 $(5 \mu \mathrm{M})$. Nos2 and Arg1 expression was assessed by qRT-PCR (A) and WB (B). (C) In vitro BM-derived MDSCs were treated for 24 hours with AT38. Nos2 and Arg1 expression were assessed by qRT-PCR. (D,E) Suppressive activity of in vitro differentiated M2-polarized macrophages (D) or MDSCs (E) treated 24 hours with AT38 was evaluated by immune suppression assay as described in methods. Cell trace dilution representative plots are shown in left panels. (F) High-performance liquid chromatography quantification of arginine and ornithine in the supernatants of in vitro generated BM-derived M0-polarized, M1-polarized, M2-polarized macrophages, treated or not for 24 hours with AT38. (G) Quantitative analysis of respiratory parameters: basal, maximal and ATP-linked respiration of in vitro generated BM-derived M0-polarized, M1-polarized, M2-polarized macrophages, treated or not for 24 hours with AT38. (H) Quantitative analysis of basal, maximal and ATP-linked respiration of in vitro differentiated CTLs treated or not with AT38 ( $5 \mu \mathrm{M}, 48$ hours). (I) Energy map displaying the overall basal metabolic profile shows a shift toward a more energetic state in AT38 treated CTLs. Data are reported as mean $\pm S D$ of three independent replicates $(D-H)$. Statistical analysis was performed using unpaired two-tailed Student's $t$-test $(A, C, F-H)$, one-way ANOVA test (D-E,G), followed by Tukey's test. ANOVA, analysis of variance; CTL, cytotoxic T lymphocyte; MDSC, myeloid-derived suppressor cell; qRT-PCR, quantitative real time-PCR. 
A
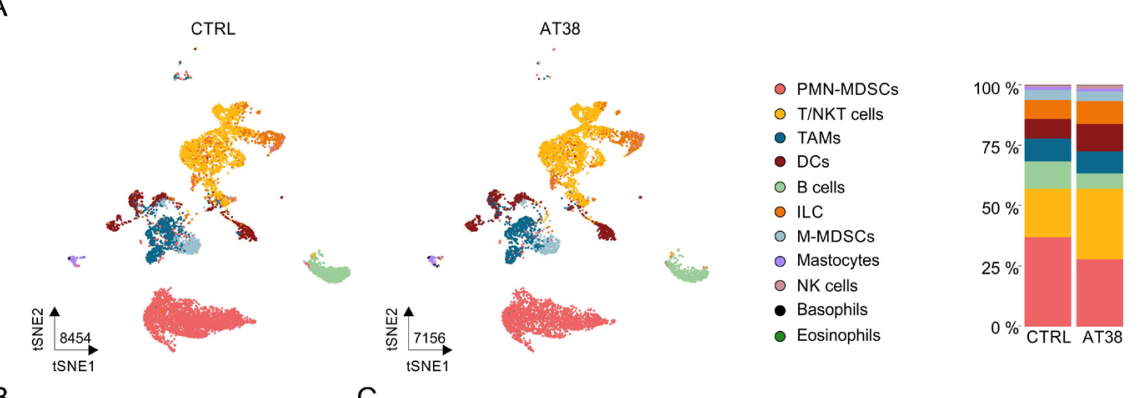

$\mathrm{B}$

C
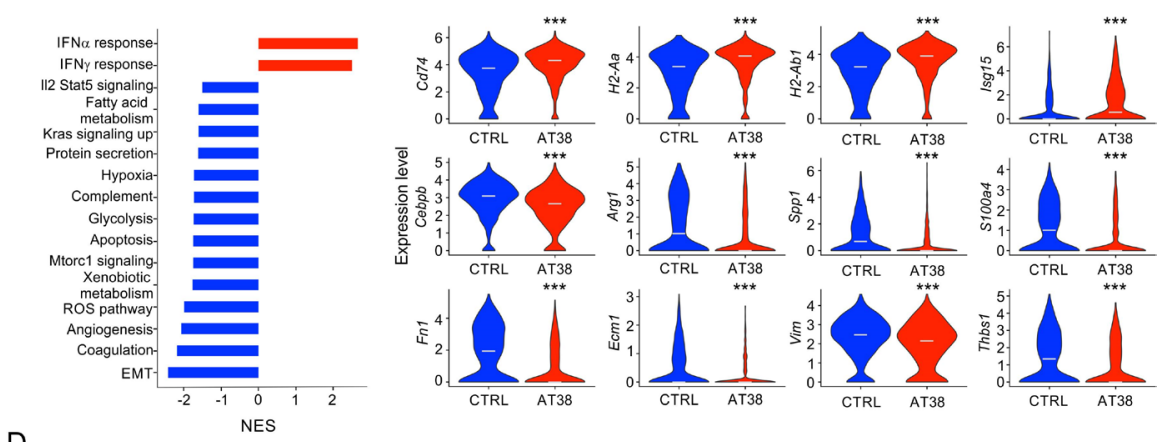

D

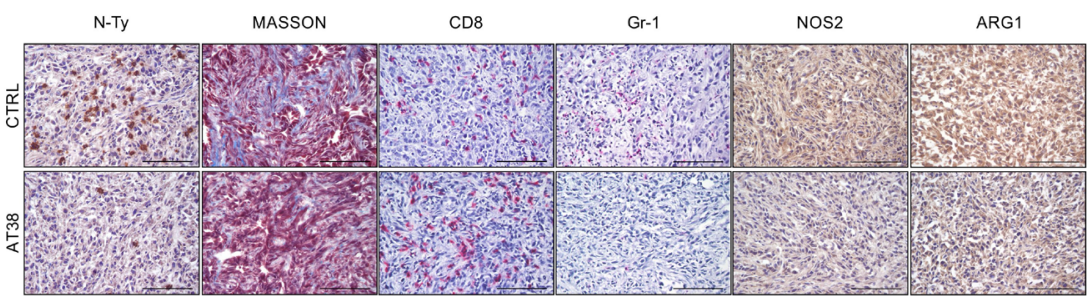

E

$\mathrm{F}$
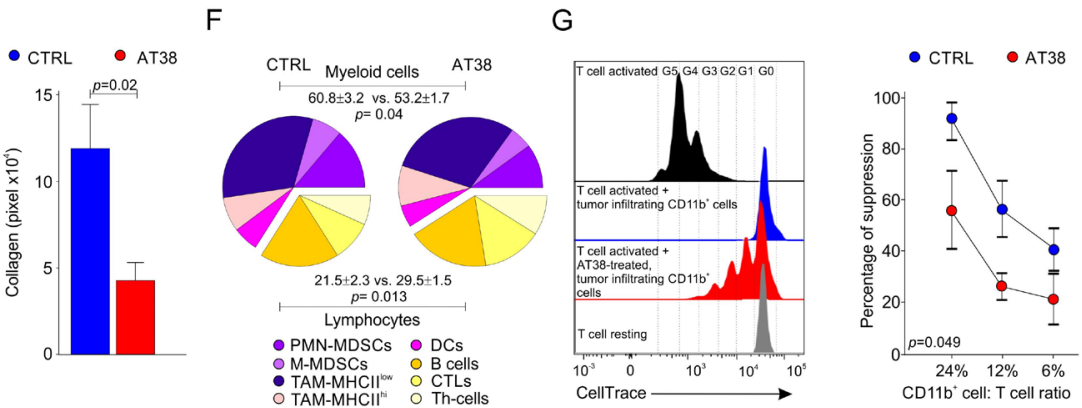

Figure 6 AT38 modifies the immune landscape of PDAC and reprograms myeloid cells toward antitumor immunity. C57BI/6 mice were orthotopically challenged with mouse KPC-derived FC1242 cells. Mice were randomized in two groups receiving $30 \mathrm{mg} / \mathrm{kg} /$ day AT38 or vehicle (CTRL) alone through a double i.p. injection each day for 7 days. Mice were sacrificed, tumors isolated and processed in order to obtain cell suspensions. Two samples/group (each one composed by a pool of four mice) were enriched for CD45 marker by FACS sorting and analyzed with scRNA-seq $10 \times$ technology as detailed in online supplemental methods. (A) UMAP representation of sCRNA-seq data for leukocytes infiltrating FC1242 tumors of CTRL and AT38 treated mice, colored according to cell types (left panel). Stacked bar plots representing cell type proportions across mouse conditions (right panel). (B) GSEA analysis with upregulated (red) or downregulated (blue) hallmark gene sets in TAMs of AT38 treated mice. (C) Violin plots showing the expression of genes involved in IFN $\alpha / \gamma$ response and antigen processing and presentation (top panel), supporting immune suppression and angiogenesis (middle panel), and promoting tumor EMT (lower panel) $\left({ }^{*} \mathrm{p}<0.05,{ }^{* *} \mathrm{p}<0.01,{ }^{* \star} \mathrm{p}<0.001\right)$ in TAMs of CTRL and AT38 treated mice. (D) Tumor samples obtained from immunocompetent mice orthotopically challenged with FC1242 tumor cells, either treated or not with AT38 for 7 days were subjected to IHC analysis to monitor RNS production (N-Ty), ECM deposition (Masson Trichrome), CTLs (CD8) and MDSCs (GR1) tumor infiltration, and the presence of NOS2 and ARG1 enzymes. Scale bar, $100 \mu m$. (E) Quantification of collagen deposition in tumor specimens of AT38-treated or untreated FC1242 tumor-bearing mice. (F) Tumor samples obtained from immunocompetent mice orthotopically challenged with FC1242 tumor cells, either treated or not with AT38 for 7 days ( $n=6$ mice/group) were subjected to multiparametric immune phenotype analysis with the following markers: B220, CD3, CD4, CD8, CD11b, CD11c, CD45, F4/80, Ly6C, Ly6G, MHCII. (G) Suppressive activity of tumor-infiltrating CD11b ${ }^{+}$cells purified from CTRL or AT38 treated mice. Data are reported as mean \pm SD of three independent replicates. Statistical analysis was performed using one-way ANOVA test. ANOVA, analysis of variance; CTL, cytotoxic T lymphocyte; IFN, interferon; NOS2, inducible nitric oxide synthase; PDAC, pancreatic ductal adenocarcinoma. 

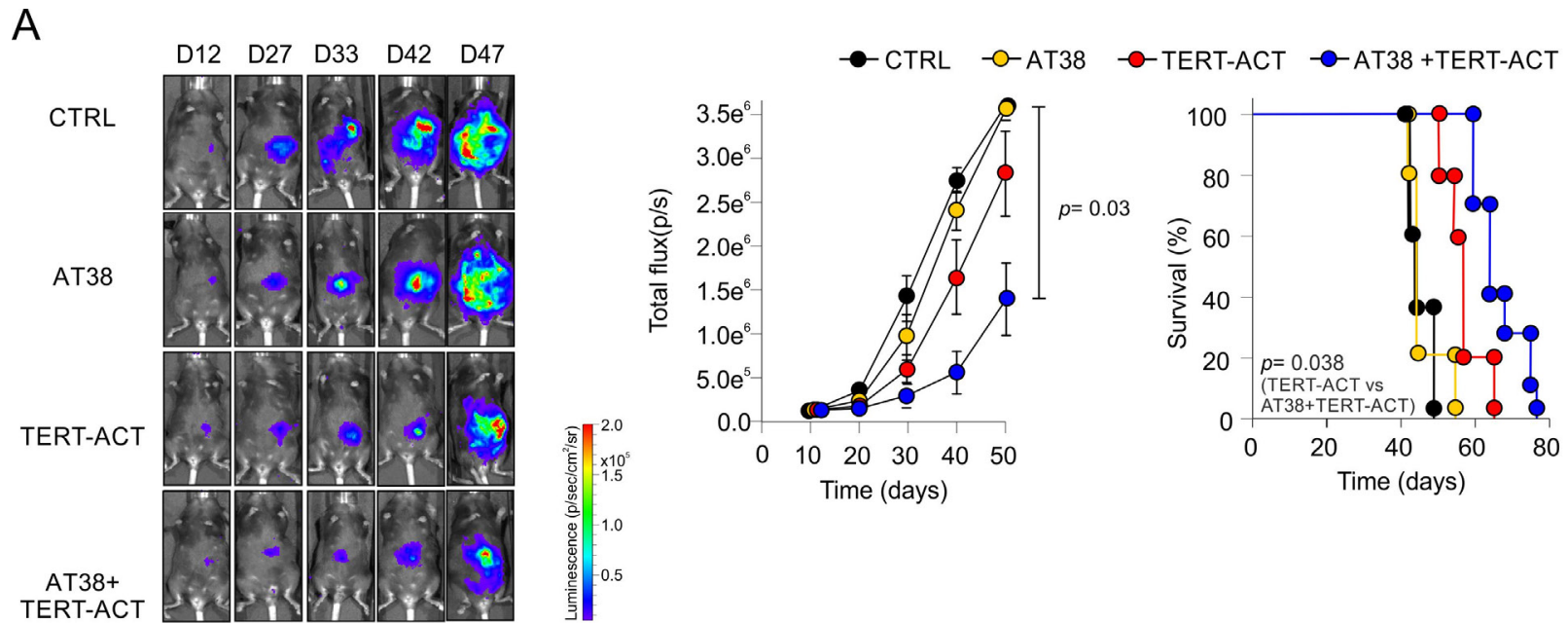

B

Change in volume $(\%)$

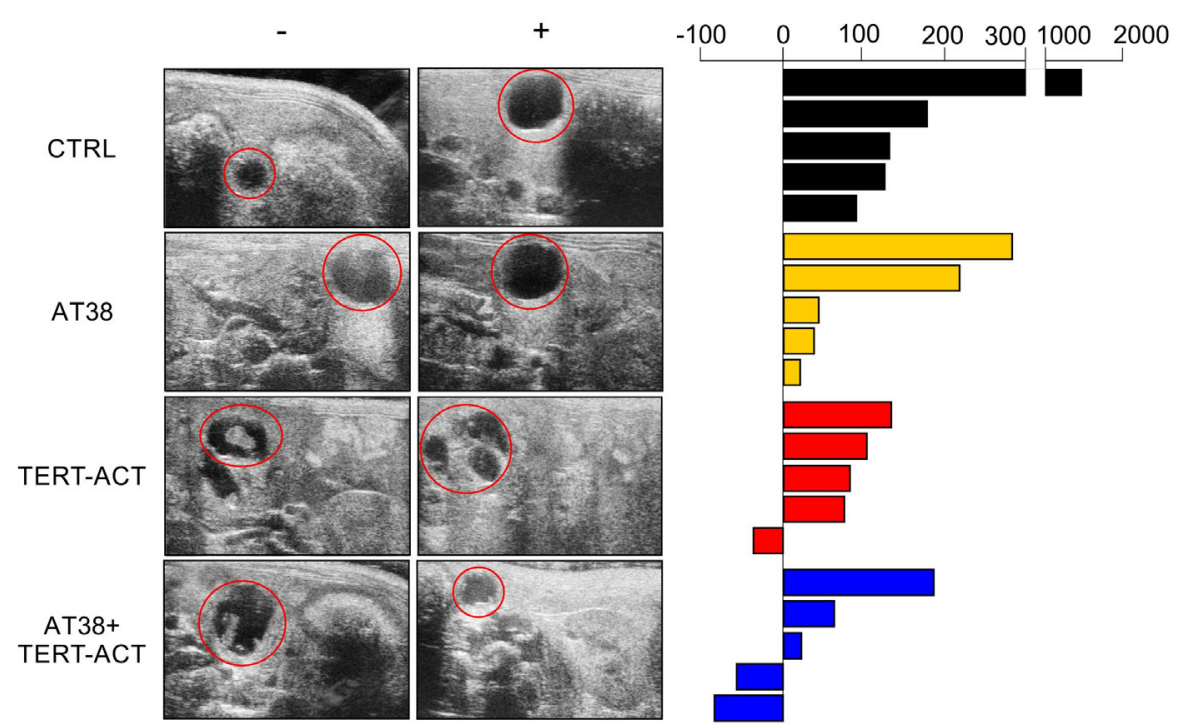

C

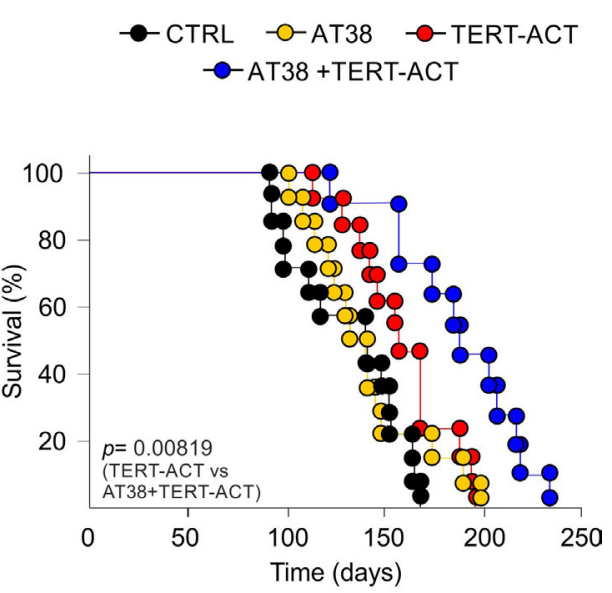

Figure 7 AT38 renders PDAC responsive to immunotherapy. (A) C57BL/6 mice were orthotopically challenged with FC1242 cells, randomized in four groups receiving, respectively, vehicle (CTRL, $n=10)$, AT38 only (AT38, $n=10$ ), $m$ TERT-specific mouse CTLs only (TERT-ACT, $n=10)$, AT38+mTERT-specific mouse CTLs $(n=10)$. Tumor growth was evaluated by in vivo imaging: a representative image for each analyzed time point was reported (left panel). Therapeutic efficacy was evaluated over time in terms of tumor progression (middle panels) and survival (right panel). Data are reported as mean $\pm S D$ of a representative experiment of two independent replicates. Statistical analysis was performed using one-way ANOVA followed by Tukey's test for tumor growth and Mantel-Haenszel (long-rank) test for survival. (B) KPC mice were enrolled in four groups receiving, respectively, vehicle (CTRL), AT38 only (AT38), mTERT-specific mouse CTLs only (TERT-ACT), AT38+mTERT-specific mouse CTLs (AT38+TERTACT). Tumor growth was evaluated by high-resolution ultrasound images of pancreatic tumor in KPC mice before (-, left panel) and 10 days after treatment (+, left panel) and shown as waterfall plots (right panel) of therapeutic response. (C) Kaplan-Meier curves for overall survival of KPC mice with invasive disease untreated $(n=25)$ or treated with AT38 $(n=20)$, TERT-ACT $(n=15)$ or combined approach $(n=20)$. Statistical analysis was performed using Mantel-Haenszel (long-rank) test. ACT, adoptive cell therapy; ANOVA, analysis of variance; CTL, cytotoxic T lymphocyte; PDAC, pancreatic ductal adenocarcinoma; TERT, telomerase.

significant increase in the survival rate (mean overall survival from 138 days for CTRL to 190 days for AT38 in combination with TERT-ACT; figure 7C).

Collectively, these results demonstrate that successful TME reprogramming by limiting RNS-dependent immune restrictions can render PDAC more susceptible to T lymphocyte-based immunotherapy.

\section{DISCUSSION}

Although immunotherapy has revolutionized the treatment of cancer, no substantial clinical benefits have been reported for PDAC when employed as a single agent. ${ }^{2}$ Several factors, including low tumor mutational burden and poor intrinsic antigenicity of PDAC cells, promote primary resistance to proficient adaptive immune 
responses induced by immune checkpoint inhibitors. Accordingly, only a small PDAC patient cohort, characterized by mismatch repair deficiency and microsatellite instability where tumors harbor neoantigens generated by mutations, showed tangible clinical responses to pembrolizumab. ${ }^{34}$ However, this proimmunogenic phenotype is quite rare in patients with PDAC, representing less than $2 \%$ of all cases. ${ }^{34}$ Therefore, only a very limited number of patients are amenable to checkpointbased therapy, suggesting the crucial need for the development of other therapeutic strategies.

Our data support the use of TERT as a target antigen for cancer immunotherapy in PDAC. We identified the TERT protein in more than $70 \%$ of human PDAC cases, confirming that TERT is a shared and leading antigen for immunotherapy. Moreover, we identified TERT expression in the tumors of a small cohort of long-term survivors, suggesting that TERT antigenicity is preserved at the late stage of PDAC evolution, and its immunogenicity might promote endogenous immune-mediated tumor control in some patients. Further studies are required to evaluate better the PDAC patients' endogenous response toward TERT antigen. Notably, TERT expression is mainly restricted to embryonic stem cells, tumor cells and, although at lower level, to adult stem cells, and male germ line. ${ }^{35-37}$ However, telomere shortening occurs during organism lifetime even in adult stem cells, confirming low TERT expression and activity in these tissues. ${ }^{36}$ In cancer, TERT gene is transcriptionally active due to the presence of two highly recurrent mutations in the gene promoter region. ${ }^{38-40}$ Accordingly, several reports have revealed the presence of anti-TERT $\mathrm{T}$ lymphocytes (absent in healthy subjects) in the blood of patients with chronic myeloid or B-cell leukemia, lung, colorectal, and breast cancers. ${ }^{11}{ }^{41}$ Owing to thymic immune-tolerance mechanisms involving the depletion of $\mathrm{T}$ lymphocytes with the highest affinity for self-proteins such as TERT and the poor T lymphocyte immunity in patients with PDAC, offthe-shelf, tumor-specific $\mathrm{T}$ lymphocyte adoptive therapy may represent an effective approach. In the present study, we report, for the first time, the possibility of restricting human and mouse PDAC progression using TERTspecific T lymphocyte ACT. These data confirm the new concept of PDAC as a 'cold' tumor and not an immune desert, suggesting the possibility of evoking an anticancer immune response via immunotherapy. Although the major limitation in developing a TERT-based therapy program for large-scale treatment is MHC-restriction, it is important to highlight two crucial aspects. First, HLA$\mathrm{A} * 0201$ represents the most frequently detected HLA-A2 allele in different ethnic groups (approximately $50 \%$ in the American and European populations). ${ }^{42}$ Second, TERT is a 'universal' antigen that is shared by $80 \%-90 \%$ of tumors with different histology. ${ }^{8}$ Therefore, a TERTbased ACT approach may be amenable to treat a very large number of patients with cancer, providing an effective solution for the treatment of orphan diseases, such as PDAC. An improved therapeutic efficacy of TERT-ACT may be obtained by exploiting the use of genetically transduced $\mathrm{T}$ memory stem cells $\left(\mathrm{T}_{\mathrm{SCM}}\right)$, which exhibit stemcell properties, high self-renewing capacity and resistance to apoptosis. Indeed, transferred genetically engineered $\mathrm{T}_{\mathrm{SCM}}$ showed an extraordinary longevity after in vivo infusion without cytokine support. ${ }^{43}$ However, potential on-target off-tumor concerns should be evaluated properly. TERT reactivation in actively proliferating cells, such as B-lymphocytes and keratinocytes, can result in the processing of the endogenous protein and antigen presentation to CTLs with high avidity, TERT-specific TCR. Mouse models cannot easily predict autoimmunity consequences of TERT-specific adoptive transfer, since TERT expression in mouse tissues is higher compared with the human counterpart. ${ }^{44}$ Species-specific difference in TERT expression can partially explain why the adoptive cell transfer of mTERT-specific CTLs are able to induce a transient, self-resolving lymphopenia. ${ }^{13}$ Conversely, human CTL clones endowed with low affinity TERT-specific TCR are not able to kill CD40-activated B cells. ${ }^{45}$ Moreover, clinical trials evaluating the safety of TERT-specific vaccines evidenced no autoimmunity side effects in patients with cancer. ${ }^{8}$

Tumors progressively establish a condition of immune resistance by promoting the expansion and accumulation of protumor cell subsets (ie, myeloid cells, CAFs, and tumor-associated endothelial cells), ${ }^{46}$ which cooperate to inhibit $\mathrm{T}$ lymphocyte fitness and trafficking inside the TME. Several reports have confirmed that T lymphocyte response in tumors is mainly abrogated by the presence of tumor-infiltrating myeloid cells. ${ }^{15}$ We showed that this paradigm is also true for PDAC. Indeed, our results revealed a shared immune sculpting of TME, in both spontaneous and implanted PDAC mouse models and clinical samples, which rely on the establishment of a chemical barrier that can shade tumors from cytotoxic T lymphocyte recognition. Accordingly, we recently proved that PMN-MDSCs and macrophage accumulation are inversely correlated with $\mathrm{T}$ lymphocyte infiltration in human pancreatic cancer specimens. ${ }^{47} \mathrm{We}$ unveiled an immunosuppressive switch, namely caspase modulator 8 and FADD-like apoptosis regulator (FLIP), whose expression in circulating monocytes (together with PDL1) is associated with worse overall survival of patients with pancreatic cancer. ${ }^{48}$ Consequently, targeting myeloid cells represents the most promising strategy for reprogramming the PDAC microenvironment. Consistent with these premises, preclinical approaches based on interfering with monocyte or granulocyte trafficking using specific blocking antibodies, ${ }^{49}$ and abrogating macrophage survival or polarization by small molecule delivery, ${ }^{50}$ have been explored. However, myeloid cell plasticity favors compensatory mechanisms, such as the proliferative rebound and expansion of untargeted cells, which limits the effectiveness of these strategies. ${ }^{25}$ CAFs also support MDSC recruitment by granulocytic chemokine release during colony stimulating factor 1 receptor (CSF1R) blockade, thereby sustaining immune-evading 
compensatory strategies. ${ }^{51}$ Altogether, these studies suggest the need for a multitargeting approach acting on more than one myeloid subset to educate a lymphocyte hostile TME toward an antitumor landscape.

The use of AT38 suits these needs. TME preconditioning with AT38 skewed the immunological landscape of pancreatic cancer in both quantitative and qualitative manners (figure 6). By integrating molecular (sc-RNA-seq), phenotypic (flow cytometry), and spatial analyses (IHC), we showed that AT38 modifies the TME immune landscape by promoting $\mathrm{T}$ lymphocyte entrance and weakening the local immune suppression established by tumor-infiltrating myeloid cells, thereby triggering an essential breach in PDAC immunotherapy resistance. Molecular analysis of tumor-infiltrating leukocytes confirmed that AT38 reprograms all myeloid compartments, inducing the activation of antitumor signaling pathways and repressing immune suppressive processes, and pathways supporting tumor angiogenesis and EMT, which are peculiar features of pro-tumor myeloid cells. Moreover, we identified increased expression of immunogenicity-associated pathways, such as antigen presentation (ie, $C d 74, H 2-A a$, and $H 2-A b 1$ in TAMs; $B 2 m$ in M-MDSCs; and Psmb8, Psmb9, and Tapbp B2m in PMNMDSCs reminiscent of tumor-associated neutrophils with antigen-presenting cell features) ${ }^{52}$, T lymphocyte stimulation, and immune recognition of cancer cells. Thus, the observed TME manipulation reconfigured TAM polarization toward an antitumor role. Although sc-RNA-seq analysis was performed only on tumor-infiltrating immune cells, we cannot exclude the possibility that AT38-dependent TME reprogramming could extend to CAFs. Accordingly, new insights have been recently added on fibroblast heterogeneity in pancreatic cancer, with the identification of a new CAF subset expressing MHCII and CD74 molecules endowed with antigen-presenting features that can activate $\mathrm{T}$ helper lymphocytes in an antigen-specific manner. ${ }^{53}$

In addition, AT38 can rewire the metabolic profile of the TME immune landscape, supporting antitumor immunity in both innate and adaptive arms. We showed that AT38 restricts ARG1 expression in both MDSCs and M2-polarized macrophages (figure 5); this pathway has a dominant hierarchical role in inducing immunosuppression by limiting arginine availability. ${ }^{55}$ Moreover, we verified that AT38 increased mitochondrial bioenergetics in CTLs (figure 5, online supplemental figure 6). Taking into account the crucial role of TME in both repressing T cell oxidative metabolism and impairing T cell fitness, ${ }^{56}$ AT38 treatment could reinvigorate dysfunctional TILs at the metabolic level. Intriguingly, a similar AT38 activity in regulating energetic metabolism of M1-polarized macrophages emerged. These cells are characterized by high production of $\mathrm{NO}$, which in turn poisons the respiratory chain and causes dependency on glycolysis. ${ }^{57-59}$ By contrast, macrophages that cannot produce NO maintain oxidative phosphorylation and mitochondrial respiratory integrity that concurrently operate with glycolysis. Therefore, AT38-dependent NOS2 abrogation can promote a metabolic reprogramming of M1-polarized macrophages supporting their antitumor abilities. The overall effect of AT38 modulation fosters an intense T-lymphocyte trafficking to the tumor core, together with an unexpected remodeling of tumor-associated stroma characterized by a significant decrease in ECM deposition (figure 6, online supplemental figure 8); all of this ultimately contributes to the improved control of tumor progression (figure 7). Notably, AT38 did not afford any clear therapeutic benefit in PDAC setting, when employed as a single treatment, suggesting no direct antitumor function (figure 7).

Our results provide a proof of concept that, by limiting nitrosative stress, tumor preconditioning may improve the efficacy of cancer immunotherapy, which is usually failing in the context of patients with pancreatic cancer. Further investigations are necessary to define patients with a peculiar, molecularly defined subset of pancreatic cancer, as well as additional tumor types and subtypes with high nitrosative stress that can benefit from this treatment. Furthermore, additional immunotherapeutic approaches might be considered in combination with AT38, such as immune checkpoint inhibitors. As nitrosative stress in the TME is peculiar to several malignancies, ${ }^{19}$ we advance that RNS-targeting by AT38 can be applied in combination with the current immune checkpoint-based protocols to increase the therapeutic success of these treatments in diverse cancer settings.

\section{CONCLUSION}

Altogether, these findings suggest that pharmacological nitrosative-stress manipulation is an effective strategy for reprogramming both the innate immune and stromal compartments of the TME to enhance immunotherapy effectiveness. In conclusion, our data strongly support the design of new, pharmacoimmunological approaches based on tumor-specific T lymphocyte ACT and drugs that render tumors more prone to immune attack. We believe that these results lay the foundation for new immunotherapy protocols for the treatment of PDAC and other solid malignancies.

\section{Author affiliations}

${ }^{1}$ Department of Medicine, Section of Immunology, University of Verona, Verona, Italy ${ }^{2}$ Department of Neurosciences, Imaging and Clinical Sciences, Center for Advanced Studies and Technnology (CAST), G. d'Annunzio University of Chieti Pescara, Chieti, Italy

${ }^{3}$ Department of Computer Science, University of Verona, Verona, Italy

${ }^{4}$ Local Health Authority of Lanciano-Vasto-Chieti, Chieti, Italy

${ }^{5}$ Pierangeli Clinic, Pescara, Italy

${ }^{6}$ Department of Medicine, University of Padua, Padova, Italy

${ }^{7}$ Veneto Institute of Oncology —Institute for Hospitalization and Care Scientific,

Padova, Italy

${ }^{8}$ Diabetes Research Institute, San Raffaele Research Centre, Milano, Italy ${ }^{9}$ School of Medicine and Surgery, Vita-Salute San Raffaele University, Milano, Italy

${ }^{10}$ Department of Diagnostic and Public Health, Section of Pharmacology, University of Verona, Verona, Italy

${ }^{11}$ General and Pancreatic Surgery Unit, University of Verona, Verona, Italy

${ }^{12}$ ARC-NET, University of Verona, Verona, Italy 
${ }^{13}$ Department of Diagnostic and Public Health, University of Verona, Verona, Italy

${ }^{14}$ Cold Spring Harbor Laboratory, Cold Spring Harbor, New York, USA

${ }^{15}$ Pancreatic Cancer Research Laboratory, Lustgarten Foundation, Cold Spring Harbor, New York, USA

\section{Twitter Francesco De Sanctis @FrancescoDS82}

Acknowledgements We thank Sara Sartori, Francesca Hofer, Varvara Petrova, and Giulio Fracasso (Immunology Section of University of Verona); Boris Rusev, Nicola Sperandio, Giada Bonizzato, and Sonia Grimaldi (ARC-Net Centre for Applied Research on Cancer) for technical assistance. We thank the Italian Foundation for Pancreatic Diseases Research ONLUS (Fondazione Italiana per la Ricerca sulle Malattie del Pancreas). We deeply acknowledge the contribution of 'Centro Piattaforme Tecnologiche' of the University of Verona for sorting and imaging experiments and 'Centro Interdipartimentale di Servizio alla Ricerca Sperimentale' of the University of Verona for maintaining mouse colonies.

Contributors FDS, SU, and VB conceived and designed the experiments. FDS and SU developed the idea. FDS, CM, RT, and SU performed animal experiments. FDS, $\mathrm{FB}$, and $\mathrm{CA}$ performed in vivo imaging. FDS, $\mathrm{AF}, \mathrm{CF}$, and SCan performed cell culture experiments. FDS, CA, and OP performed molecular experiments. FDS, CM, and TC performed cell-sorting experiments. SCal and RG performed all the bioinformatics analysis. ALA, FDP, and MI performed histology and immunohistochemistry analysis of all mouse samples and TME characterization in human samples. MF performed histology and immunohistochemistry analysis of TERT in human samples. ED and LP provided mouse samples for histology analysis. EB and ID performed seahorse studies and interpreted extracellular flux data. SP, RS, RTL, CB and AS organized the entire patient study, collected the samples and analyzed the clinical data. GDR, BZ, and MI analyzed human pancreatic samples for TME study. YP and DT provided KPC-derived cell lines and experimental mouse models. VC, MF, AS, and Ml interpreted histological data; FDS, SU, and VB interpreted the data. SS assisted with the manuscript writing; FDS, SU, and VB wrote the manuscript. SU and VB supervised the project and are guarantors of the study. All the authors have read and approved the manuscript.

Funding This work was jointly supported by VB grants $(23788,12182)$ that refer to Special Program Molecular Clinical Oncology 5 per mille) and fellowships (Francesco De Sanctis, AIRC/FIRC 2013, Alessandra Fiore, AIRC/FIRC 2017 and Rosalinda Trovato, AIRC/FIRC 2018) from Italian Association for Cancer Research (AIRC) and by grants provided by: the Cancer Research Institute (Clinic and Laboratory Integration Program, CLIP_2020), Euronanomed III (Joint Translational Call_2017, Project Resolve), Cariverona Foundation (Project call, 2017) and PRIN program of Italian Ministry of Education, University and Research (MIUR, CUP: B38D19000260006) as well as by US grants provided by AIRC (MFAG project: 21509) and PRIN program of MIUR (CUP: B38D19000140006). This work was also supported by LP grant from Italian association for cancer research (AIRC 22737), CB grant (RF-2016-02361940) from University of Verona. VC is supported by AIRC (Grant N 18718). DT is supported by the NIH Cancer Center Support Grant (5P30CA045508) and the Lustgarten Foundation, where he is a distinguished scholar, Director of the Lustgarten Foundation-designated Laboratory of Pancreatic Cancer Research and Director of CSHL Cancer Center. YP is supported by NCl R50CA211506.

Competing interests $\mathrm{AF}, \mathrm{SU}$, and VB hold proprietary rights on the patent applications about engineered cells for inducing tolerance by BioNTech (Mainz, Germany). SU and VB hold proprietary rights on the patent applications about TERT engineering T cells by University of Verona (Verona, Italy). VB holds proprietary rights on the patent applications about nitric oxide furoxan derivative compounds by Humanitas Mirasole (Milan, Italy). MF reports grants from Astellas Pharma, QED Therapeutics, Macrophage Pharma and advisory roles in Astellas Pharma, Tesaro, GlaxoSmithKline, Diaceutics, Roche. VB reports personal fees from Codiak BioSciences and IO Biotech ApS outside the submitted work. No potential conflicts of interest were disclosed by the other authors.

\section{Patient consent for publication Not applicable.}

Ethics approval This study involves human participants. For the use of PDAC and HD samples, informed consent was obtained from all participants and the study was approved by the Ethics Committee: Prot. 25978, Prog. 2172 on 29/05/2012 from Comitato Etico per la sperimentazione clinica delle province di Verona e Rovigo; Principal investigator: Aldo Scarpa. Participants gave informed consent to participate in the study before taking part.

Provenance and peer review Not commissioned; externally peer reviewed.
Data availability statement Sequencing data are available in a public, open access repository. Single-cell RNA-sequencing analysis of tumor-infiltrating leukocytes isolated from or AT38-treated or untreated pancreatic tumor-bearing mice have been deposited at NCBI's Gene Expression Omnibus and are accessible under GEO Series accession number GSE179273.

Supplemental material This content has been supplied by the author(s). It has not been vetted by BMJ Publishing Group Limited (BMJ) and may not have been peer-reviewed. Any opinions or recommendations discussed are solely those of the author(s) and are not endorsed by BMJ. BMJ disclaims all liability and responsibility arising from any reliance placed on the content. Where the content includes any translated material, BMJ does not warrant the accuracy and reliability of the translations (including but not limited to local regulations, clinical guidelines, terminology, drug names and drug dosages), and is not responsible for any error and/or omissions arising from translation and adaptation or otherwise.

Open access This is an open access article distributed in accordance with the Creative Commons Attribution Non Commercial (CC BY-NC 4.0) license, which permits others to distribute, remix, adapt, build upon this work non-commercially, and license their derivative works on different terms, provided the original work is properly cited, appropriate credit is given, any changes made indicated, and the use is non-commercial. See http://creativecommons.org/licenses/by-nc/4.0/.

\section{ORCID iDs}

Chiara Musiu http://orcid.org/0000-0003-1647-5708

Vincenzo Bronte http://orcid.org/0000-0002-3741-5141

\section{REFERENCES}

1 Rahib L, Smith BD, Aizenberg R, et al. Projecting cancer incidence and deaths to 2030: the unexpected burden of thyroid, liver, and pancreas cancers in the United States. Cancer Res 2014;74:2913-21.

2 Brahmer JR, Tykodi SS, Chow LQM, et al. Safety and activity of anti-PD-L1 antibody in patients with advanced cancer. $N$ Engl J Med 2012;366:2455-65

3 Lawrence MS, Stojanov P, Polak P, et al. Mutational heterogeneity in cancer and the search for new cancer-associated genes. Nature 2013;499:214-8

4 Ho WJ, Jaffee EM, Zheng L. The tumour microenvironment in pancreatic cancer - clinical challenges and opportunities. Nat Rev Clin Oncol 2020;17:527-40.

5 Hingorani SR, Wang L, Multani AS, et al. Trp53R172H and KrasG12D cooperate to promote chromosomal instability and widely metastatic pancreatic ductal adenocarcinoma in mice. Cancer Cell 2005;7:469-83.

6 McGranahan N, Furness AJS, Rosenthal R, et al. Clonal neoantigens elicit $\mathrm{T}$ cell immunoreactivity and sensitivity to immune checkpoint blockade. Science 2016;351:1463-9.

7 Amedei A, Niccolai E, Prisco D. Pancreatic cancer: role of the immune system in cancer progression and vaccine-based immunotherapy. Hum Vaccin Immunother 2014;10:3354-68.

8 Zanetti M. A second chance for telomerase reverse transcriptase in anticancer immunotherapy. Nat Rev Clin Oncol 2017;14:115-28.

9 Hanahan D, Weinberg RA. Hallmarks of cancer: the next generation. Cell 2011;144:646-74.

10 Mennuni C, Ugel S, Mori F, et al. Preventive vaccination with telomerase controls tumor growth in genetically engineered and carcinogen-induced mouse models of cancer. Cancer Res 2008;68:9865-74.

11 Sandri S, Bobisse S, Moxley K, et al. Feasibility of telomerasespecific adoptive T-cell therapy for B-cell chronic lymphocytic leukemia and solid malignancies. Cancer Res 2016;76:2540-51.

12 Sandri S, De Sanctis F, Lamolinara A, et al. Effective control of acute myeloid leukaemia and acute lymphoblastic leukaemia progression by telomerase specific adoptive T-cell therapy. Oncotarget 2017;8:86987-7001.

13 Ugel S, Scarselli E, lezzi M, et al. Autoimmune B-cell lymphopenia after successful adoptive therapy with telomerase-specific $\mathrm{T}$ lymphocytes. Blood 2010;115:1374-84.

14 Peng J, Sun B-F, Chen C-Y, et al. Single-cell RNA-seq highlights intra-tumoral heterogeneity and malignant progression in pancreatic ductal adenocarcinoma. Cell Res 2019;29:725-38.

15 Sharma P, Hu-Lieskovan S, Wargo JA, et al. Primary, adaptive, and acquired resistance to cancer immunotherapy. Cell 2017;168:707-23.

16 Bayne LJ, Beatty GL, Jhala N, et al. Tumor-derived granulocytemacrophage colony-stimulating factor regulates myeloid 
inflammation and T cell immunity in pancreatic cancer. Cancer Cell 2012;21:822-35.

17 Ugel S, Canè S, De Sanctis F, et al. Monocytes in the tumor microenvironment. Annu Rev Pathol 2021;16:93-122.

18 De Sanctis F, Sandri S, Ferrarini G, et al. The emerging immunological role of post-translational modifications by reactive nitrogen species in cancer microenvironment. Front Immunol 2014;5:69.

19 Molon B, Ugel S, Del Pozzo F, et al. Chemokine nitration prevents intratumoral infiltration of antigen-specific T cells. J Exp Med 2011;208:1949-62.

20 Hiyama E, Kodama T, Shinbara K, et al. Telomerase activity is detected in pancreatic cancer but not in benign tumors. Cancer Res 1997;57:326-31.

21 Dugnani E, Pasquale V, Marra P, et al. Four-class tumor staging for early diagnosis and monitoring of murine pancreatic cancer using magnetic resonance and ultrasound. Carcinogenesis 2018;39:1197-206.

22 Klebanoff CA, Gattinoni L, Palmer DC, et al. Determinants of successful CD8+ T-cell adoptive immunotherapy for large established tumors in mice. Clin Cancer Res 2011;17:5343-52.

23 O'Donnell JS, Teng MWL, Smyth MJ. Cancer immunoediting and resistance to T cell-based immunotherapy. Nat Rev Clin Oncol 2019;16:151-67.

24 Beatty GL, Winograd R, Evans RA, et al. Exclusion of T cells from pancreatic carcinomas in mice is regulated by Ly6C(low) F4/80(+) extratumoral macrophages. Gastroenterology 2015;149:201-10.

25 Stromnes IM, Brockenbrough JS, Izeradjene K, et al. Targeted depletion of an MDSC subset unmasks pancreatic ductal adenocarcinoma to adaptive immunity. Gut 2014;63:1769-81.

26 Noy R, Pollard JW. Tumor-associated macrophages: from mechanisms to therapy. Immunity 2014;41:49-61.

27 Bear AS, Vonderheide RH, O'Hara MH. Challenges and opportunities for pancreatic cancer immunotherapy. Cancer Cell 2020;38:788-802.

28 Marigo I, Zilio S, Desantis G, et al. T cell cancer therapy requires CD40-CD40L activation of tumor necrosis factor and inducible nitric-oxide-synthase-producing dendritic cells. Cancer Cell 2016;30:377-90.

29 Pereira PMR, Edwards KJ, Mandleywala K, et al. iNOS regulates the therapeutic response of pancreatic cancer cells to radiotherapy. Cancer Res 2020;80:1681-92.

30 Liudahl SM, Betts CB, Sivagnanam S, et al. Leukocyte heterogeneity in pancreatic ductal adenocarcinoma: phenotypic and spatial features associated with clinical outcome. Cancer Discov 2021:11:2014-31.

31 Cassetta L, Pollard JW. Targeting macrophages: therapeutic approaches in cancer. Nat Rev Drug Discov 2018;17:887-904.

32 De Sanctis F, Solito S, Ugel S, et al. MDSCs in cancer: conceiving new prognostic and therapeutic targets. Biochim Biophys Acta 2016;1865:35-48.

33 Szabó C, Ischiropoulos H, Radi R. Peroxynitrite: biochemistry, pathophysiology and development of therapeutics. Nat Rev Drug Discov 2007;6:662-80

34 Le DT, Durham JN, Smith KN, et al. Mismatch repair deficiency predicts response of solid tumors to PD-1 blockade. Science 2017:357:409-13.

35 Liu L, Bailey SM, Okuka M, et al. Telomere lengthening early in development. Nat Cell Biol 2007:9:1436-41.

36 Flores I, Benetti R, Blasco MA. Telomerase regulation and stem cell behaviour. Curr Opin Cell Biol 2006;18:254-60.

37 Flores I, Cayuela ML, Blasco MA. Effects of telomerase and telomere length on epidermal stem cell behavior. Science 2005;309:1253-6.

38 Horn S, Figl A, Rachakonda PS, et al. TERT promoter mutations in familial and sporadic melanoma. Science 2013;339:959-61.

39 Huang FW, Hodis E, Xu MJ, et al. Highly recurrent TERT promoter mutations in human melanoma. Science 2013;339:957-9.

40 Vinagre J, Almeida A, Pópulo H, et al. Frequency of TERT promoter mutations in human cancers. Nat Commun 2013;4:2185.
41 Filaci G, Fravega M, Setti M, et al. Frequency of telomerasespecific CD8+ T lymphocytes in patients with cancer. Blood 2006;107:1505-12.

42 Ellis JM, Henson V, Slack R, et al. Frequencies of HLA-A2 alleles in five U.S. population groups. predominance of $A^{*} 02011$ and identification of HLA-A*0231. Hum Immunol 2000;61:334-40.

43 Biasco L, Scala S, Basso Ricci L, et al. In vivo tracking of T cells in humans unveils decade-long survival and activity of genetically modified T memory stem cells. Sci Transl Med 2015;7:273ra13.

44 Martín-Rivera L, Herrera E, Albar JP, et al. Expression of mouse telomerase catalytic subunit in embryos and adult tissues. Proc Natl Acad Sci U S A 1998;95:10471-6.

45 Hernandez J, Garcia-Pons F, Lone YC, et al. Identification of a human telomerase reverse transcriptase peptide of low affinity for HLA A2.1 that induces cytotoxic T lymphocytes and mediates lysis of tumor cells. Proc Natl Acad Sci U S A 2002;99:12275-80.

46 De Sanctis F, Ugel S, Facciponte J, et al. The dark side of tumorassociated endothelial cells. Semin Immunol 2018;35:35-47.

47 Trovato R, Fiore A, Sartori S, et al. Immunosuppression by monocytic myeloid-derived suppressor cells in patients with pancreatic ductal carcinoma is orchestrated by STAT3. $\mathrm{J}$ Immunother Cancer 2019;7:255

48 Fiore A, Ugel S, De Sanctis F, et al. Induction of immunosuppressive functions and NF-kappaB by FLIP in monocytes. Nat Commun 2018;9:5193.

49 Nywening TM, Belt BA, Cullinan DR, et al. Targeting both tumourassociated $\mathrm{CXCR} 2^{+}$neutrophils and $\mathrm{CCR} 2^{+}$macrophages disrupts myeloid recruitment and improves chemotherapeutic responses in pancreatic ductal adenocarcinoma. Gut 2018;67:1112-23.

50 Zhu Y, Knolhoff BL, Meyer MA, et al. CSF1/CSF1R blockade reprograms tumor-infiltrating macrophages and improves response to T-cell checkpoint immunotherapy in pancreatic cancer models. Cancer Res 2014;74:5057-69.

51 Kumar V, Donthireddy L, Marvel D, et al. Cancer-associated fibroblasts neutralize the anti-tumor effect of CSF1 receptor blockade by inducing PMN-MDSC infiltration of tumors. Cancer Cell 2017;32:654-68.

52 Singhal S, Bhojnagarwala PS, O'Brien S, et al. Origin and role of a subset of tumor-associated neutrophils with antigen-presenting cell features in early-stage human lung cancer. Cancer Cell 2016;30:120-35.

53 Elyada E, Bolisetty M, Laise P, et al. Cross-species single-cell analysis of pancreatic ductal adenocarcinoma reveals antigenpresenting cancer-associated fibroblasts. Cancer Discov 2019;9:1102-23.

54 Van de Velde L-A, Subramanian C, Smith AM, et al. T cells encountering myeloid cells programmed for amino acid-dependent immunosuppression use Rictor/mTORC2 protein for proliferative checkpoint decisions. J Biol Chem 2017;292:15-30.

55 Marigo I, Bosio E, Solito S, et al. Tumor-induced tolerance and immune suppression depend on the C/EBPbeta transcription factor. Immunity 2010;32:790-802.

56 Scharping NE, Menk AV, Moreci RS, et al. The tumor microenvironment represses $\mathrm{T}$ cell mitochondrial biogenesis to drive intratumoral T cell metabolic insufficiency and dysfunction. Immunity 2016;45:374-88.

57 Everts B, Amiel E, van der Windt GJW, et al. Commitment to glycolysis sustains survival of NO-producing inflammatory dendritic cells. Blood 2012;120:1422-31.

58 Palmieri EM, Gonzalez-Cotto M, Baseler WA, et al. Nitric oxide orchestrates metabolic rewiring in M1 macrophages by targeting aconitase 2 and pyruvate dehydrogenase. Nat Commun 2020;11:698.

59 McNeill E, Stylianou E, Crabtree MJ, et al. Regulation of mycobacterial infection by macrophage $\mathrm{GCH} 1$ and tetrahydrobiopterin. Nat Commun 2018;9:5409. 